\title{
Adaptive Finite Element Method for Optimal Control Problem Governed by Linear Quasiparabolic Integrodifferential Equations
}

\author{
Wanfang Shen ${ }^{1,2}$ and Hua Su${ }^{2}$ \\ ${ }^{1}$ School of Mathematics, Shandong University, Jinan 250100, China \\ ${ }^{2}$ School of Mathematic and Quantitative Economics, Shandong University of Finance and Economics, \\ Jinan 250014, China \\ Correspondence should be addressed to Wanfang Shen, wanfangshen@gmail.com
}

Received 21 June 2012; Accepted 10 July 2012

Academic Editor: Xinguang Zhang

Copyright (c) 2012 W. Shen and H. Su. This is an open access article distributed under the Creative Commons Attribution License, which permits unrestricted use, distribution, and reproduction in any medium, provided the original work is properly cited.

The mathematical formulation for a quadratic optimal control problem governed by a linear quasiparabolic integrodifferential equation is studied. The control constrains are given in an integral sense: $U_{a d}=\left\{u \in X ; \int_{\Omega_{U}} u \geqslant 0, t \in[0, T]\right\}$. Then the a posteriori error estimates in $L^{\infty}\left(0, T ; H^{1}(\Omega)\right)$-norm and $L^{2}\left(0, T ; L^{2}(\Omega)\right)$-norm for both the state and the control approximation are given.

\section{Introduction}

Integrodifferential equations of quasiparabolic and their control of this nature appear in applications such as biology mechanics, nuclear reaction dynamics, heat conduction in materials with memory, and viscoelasticity. All these models express a conservation of a certain quantity in any moment for any subdomain and the historical accumulation feature in the physical models. This in many applications is the most desirable feature of the approximation method when it comes to numerical solution of the corresponding initial boundary value problem. The existence and uniqueness of the solution of the quasiparabolic Integrodifferential equations has been studied in [1]. Finite element methods for quasiparabolic Integrodifferential equations problems with a smooth kernel have been discussed in Cui [2]. Although there is so much work for the finite element approximation of this problem, to our knowledge, there has been a lack of a posteriori error estimates for finite element approximation of any quasiparabolic Integrodifferential optimal control problem. 
The finite element approximation of optimal control problems has been an important topic in engineering design works. There have been extensive theoretical and numerical studies for various optimal control problems, see, for instance, [3-11], although it is impossible to give even a very brief review here. And research on finite element approximation of parabolic optimal control problems can be found in, for example, [12, 13].

Among many finite element methods, the adaptive finite element method based on a posteriori error estimates has become a central theme in scientific and engineering computations for its high efficiency. In order to obtain a numerical solution of acceptable accuracy, it is essential for the adaptive finite element method to use a posteriori error estimate indicators to guide the mesh refinement procedure. We only need refine the area where the error indicators are larger, so that a higher density of nodes are distributed over the area where the solution is difficult to approximate. In this sense, adaptive finite element approximation relies very much on the error indicators used, which are often based on a posteriori error estimates of the solutions.

The purpose of this paper is to derive the a posteriori error estimates for the semidiscrete finite element approximation of a quadratic optimal control problem governed by a linear quasiparabolic Integrodifferential equation, which paves a way to derive the a posteriori error estimates for the full discrete finite element approximation for this control problem and thus to develop its adaptive finite element schemes. We extend the existing techniques and results in [14-16] to the optimal control problem governed by the Integrodifferential equation of quasiparabolic type.

The outline of the paper is as follows. In Section 2, we first briefly introduce the optimal control problem and give the optimality conditions, then construct the finite element approximation schemes for the optimal control problem. In Section 3, we give the a posteriori error bounds in $L^{\infty}\left(0, T ; H^{1}(\Omega)\right)$-norm for the control problem. And the a posteriori error bounds in $L^{2}\left(0, T ; L^{2}(\Omega)\right)$-norm for the control problem are derived in Section 4.

\section{Optimal Control Problem and Its Finite Element Approximation}

Let $\Omega$ and $\Omega_{U}$ be bounded convex polygon domains in $R^{d}$ with Lipschitz boundary $\partial \Omega$ and $\partial \Omega_{U}$. In this paper, we adopt the standard notation $W^{m, q}(\Omega)$ for Sobolev spaces on $\Omega$ with norm $\|\cdot\|_{m, q, \Omega}$, and seminorm $|\cdot|_{m, q, \Omega}$. We set $W_{0}^{m, q}(\Omega)=\left\{w \in W^{m, q}(\Omega):\left.w\right|_{\partial \Omega}=0\right\}$. We denote $W^{m, 2}(\Omega)\left(W_{0}^{m, 2}(\Omega)\right)$ by $H^{m}(\Omega)\left(H_{0}^{m}(\Omega)\right)$, with norm $\|\cdot\|_{m, \Omega}$, and seminorm $|\cdot|_{m, \Omega}$.

We denote by $L^{s}\left(0, T ; W^{m, q}(\Omega)\right)$ the Banach space of all $L^{s}$ integrable functions from $(0, T)$ into $W^{m, q}(\Omega)$ with norm $\|v\|_{L^{s}\left(0, T ; W^{m, q}(\Omega)\right)}=\left(\int_{0}^{T}\|v\|_{W^{m, q}(\Omega)}^{s} d t\right)^{1 / s}$ for $s \in[1, \infty)$ and the standard modification for $s=\infty$. Similarly, one can define the spaces $H^{1}\left(0, T ; W^{m, q}(\Omega)\right)$ and $C^{k}\left(0, T ; W^{m, q}(\Omega)\right)$. The details can be found in [17]. In addition, $c$ or $C$ denotes a general positive constant independent of the mesh size $h$.

In the following, we will give semi-discrete finite element approximation schemes for the optimal control problem governed by a linear quasiparabolic Integrodifferential equation.

\subsection{Model Problem and Its Weak Formulation}

We will take the state space $W=L^{2}(0, T ; V)$ with $V=H_{0}^{1}(\Omega)$ and the control space $X=$ $L^{2}(0, T ; U)$ with $U=L^{2}\left(\Omega_{U}\right)$. Let the observation space $Y=L^{2}(0, T ; H)$ with $H=L^{2}(\Omega)$ and $U_{a d} \subseteq X$ a convex subset. 
We are interested in the following optimal control problem:

$$
\min _{u \in U_{a d} \subset X} J(u, y(u))=\frac{1}{2}\left\{\int_{0}^{T}\left\|y-z_{d}\right\|_{0, \Omega}^{2} d t+\int_{0}^{T}\|u\|_{0, \Omega_{u}}^{2} d t\right\},
$$

subject to

$$
\begin{gathered}
y_{t}-\operatorname{div}\left(A \nabla y_{t}+D \nabla y+\int_{0}^{t} C(t, \tau) \nabla y(x, \tau) d \tau\right)=f+B u, \quad \text { in } \Omega \times(0, T], \\
y=0, \quad \text { on } \partial \Omega \times[0, T] \\
\left.y\right|_{t=0}=y^{0}, \quad \text { in } \Omega,
\end{gathered}
$$

where $u$ is control, $y$ is state, $z_{d}$ is the observation, $U_{a d}$ is a closed convex subset, $f(x, t) \in$ $L^{2}\left(0, T ; L^{2}(\Omega)\right)$, and $z_{d}$ and $y^{0} \in H^{1}(\Omega)$ are some suitable functions to be specified later. $B$ is a linear bounded operator from $L^{2}\left(\Omega_{U}\right)$ to $L^{2}(\Omega)$ independent of $t$. And

$$
A=A(x)=\left(a_{i, j}(\cdot)\right)_{n \times n} \in\left(C^{\infty}(\bar{\Omega})\right)^{n \times n}, \quad D=D(x)=\left(d_{i, j}(\cdot)\right)_{n \times n} \in\left(C^{\infty}(\bar{\Omega})\right)^{n \times n},
$$

such that there is a constant $c>0$ satisfying that for any vector $X \in R^{n}$ as follows:

$$
X^{t} A X \geq c\|X\|_{R^{n}}^{2}, \quad X^{t} D X \geq c\|X\|_{R^{n}}^{2},
$$

$C=C(x, t, \tau)=\left(c_{i, j}(x, t, \tau)\right)_{n \times n} \in\left(C^{\infty}\left(0, T ; L^{2}(\bar{\Omega})\right)^{n \times n}\right)$.

Let

$$
\begin{gathered}
\left(f_{1}, f_{2}\right)=\int_{\Omega} f_{1} f_{2}, \quad \forall\left(f_{1}, f_{2}\right) \in H \times H, \\
(u, v)_{U}=\int_{\Omega_{U}} u v, \quad \forall(u, v) \in U \times U, \\
a(z, \omega)=(A \nabla z, \nabla \omega), \quad d(z, \omega)=(D \nabla z, \nabla \omega), \\
c(t, \tau ; z, \omega)=(C(t, \tau) \nabla z, \nabla \omega), \quad \forall z, w \in V \times V .
\end{gathered}
$$

In the case that $f_{1} \in V$ and $f_{2} \in V^{*}$, the dual pair $\left(f_{1}, f_{2}\right)$ is understood as $\left\langle f_{1}, f_{2}\right\rangle_{V \times V^{*}}$.

Assume that there are constants $c$ and $C$, such that for all $t$ and $\tau$ in $[0, T]$ as follows:

$$
\begin{aligned}
& \text { (a) } a(z, z) \geqslant c\|z\|_{1, \Omega}^{2} \\
& \text { (b) }|a(z, w)| \leqslant C\|z\|_{1, \Omega}\|w\|_{1, \Omega}, \quad|d(z, w)| \leqslant C\|z\|_{1, \Omega}\|w\|_{1, \Omega}, \\
& \text { (c) }|c(t, \tau ; z, w)| \leqslant C\|z\|_{1, \Omega}\|w\|_{1, \Omega} .
\end{aligned}
$$

for any $z$ and $w$ in $V$. 
Then the weak form of the state equation reads as

$$
\begin{gathered}
\left(y_{t}, w\right)+a\left(y_{t}, w\right)+d(y, w)+\int_{0}^{t} c(t, \tau ; y(\tau), w) d \tau=(f+B u, w) \\
\forall w \in V, t \in(0, T], \\
\left.y\right|_{t=0}=y^{0} .
\end{gathered}
$$

It is well known (see, e.g., [1]) that the above weak formulation has at least one solution in $y \in W(0, T)=\left\{w \in L^{\infty}\left(0, T ; H^{1}(\Omega)\right), w_{t}^{\prime} \in L^{2}\left(0, T ; H^{1}(\Omega)\right)\right\}$.

Therefore, the weak form of the control problem (2.1) and (2.2) reads as (OCP)

$$
\begin{gathered}
\min _{u \in U_{a d}} J(u, y(u)), \\
\left(y_{t}, w\right)+a\left(y_{t}, w\right)+d(y, w)+\int_{0}^{t} c(t, \tau ; y(\tau), w) d \tau=(f+B u, w) \quad \forall w \in V, t \in(0, T], \\
\left.y\right|_{t=0}=y^{0} .
\end{gathered}
$$

In the following, we first give the existence and uniqueness of the solution of the system (2.8).

Theorem 2.1. Assume that the condition (2.6) (a)-(c) holds. There exists the unique solution $(u, y)$ for the minimization problem (2.8) such that $u \in L^{2}\left(0, T ; L^{2}\left(\Omega_{U}\right)\right), y \in L^{\infty}\left(0, T ; H^{1}(\Omega)\right)$, and $y_{t} \in L^{2}\left(0, T ; H^{1}(\Omega)\right)$.

Proof. Let $\left\{\left(u^{n}, y^{n}\right)\right\}_{n=1}^{\infty}$ be a minimization sequence for the system (2.8), then the sequence $\left\{u^{n}\right\}_{n=1}^{\infty}$ is bounded in $L^{2}\left(0, T ; L^{2}\left(\Omega_{U}\right)\right)$. Thus there is a subsequence of $\left\{u^{n}\right\}_{n=1}^{\infty}$ (still denote by $\left.\left\{u^{n}\right\}_{n=1}^{\infty}\right)$ such that $u^{n}$ converges to $u^{*}$ weakly in $L^{2}\left(0, T ; L^{2}\left(\Omega_{U}\right)\right)$. For the subsequence $\left\{u^{n}\right\}_{n=1}^{\infty}$, we have

$$
\begin{aligned}
& \left(y_{t}^{n}, w\right)+a\left(y_{t}^{n}, w\right)+d\left(y^{n}, w\right)+\int_{0}^{t} c\left(t, \tau ; y^{n}(\tau), w(t)\right) d \tau=\left(f+B u^{n}, w\right) \\
& \forall w \in V, t \in(0, T] .
\end{aligned}
$$

By setting $w=y^{n}$ and integrating from 0 to $t$ in (2.9), we give

$$
\begin{aligned}
& \left\|y^{n}(t)\right\|_{1, \Omega}^{2}+\int_{0}^{t}\left\|y^{n}\right\|_{1, \Omega}^{2} d \tau \\
& \quad \leqslant C\left\{\left\|y^{0}\right\|_{1, \Omega}+C \int_{0}^{t}\left(\|f\|_{-1, \Omega}^{2}+\left\|u^{n}\right\|_{0, \Omega u}^{2}\right) d t+\int_{0}^{t} \int_{0}^{\tau}\|y(s)\|_{1, \Omega}^{2} d s d \tau\right\} .
\end{aligned}
$$

Applying Gronwall's inequality to (2.10) yields

$$
\left\|y^{n}\right\|_{L^{\infty}\left(0, T ; H^{1}(\Omega)\right)}^{2}+\left\|y^{n}\right\|_{L^{2}\left(0, T ; H^{1}(\Omega)\right)}^{2} \leqslant C\left\{\left\|y^{0}\right\|_{1, \Omega}^{2}+\int_{0}^{T}\left(\|f\|_{-1, \Omega}^{2}+\left\|u^{n}\right\|_{0, \Omega_{U}}^{2}\right)\right\} .
$$


So $\left\{u^{n}\right\}_{n=1}^{\infty}$ is a bounded set in $L^{2}\left(0, T ; L^{2}\left(\Omega_{U}\right)\right)$ and $\left\{y^{n}\right\}_{n=1}^{\infty}$ is a bounded set in $L^{\infty}(0, T$; $H^{1}(\Omega)$ ). Thus

$$
\begin{gathered}
u^{n} \longrightarrow u \text { weakly in } L^{2}\left(0, T ; L^{2}\left(\Omega_{U}\right)\right), \\
y^{n} \longrightarrow y \quad \text { weakly in } L^{\infty}\left(0, T ; H^{1}(\Omega)\right), \\
y^{n}(T) \longrightarrow y(T) \quad \text { weakly in } H^{1}(\Omega) .
\end{gathered}
$$

Let $W=\left\{w ; w \in L^{\infty}\left(0, T ; H^{1}(\Omega)\right), w_{t}^{\prime} \in L^{2}\left(0, T ; H^{1}(\Omega)\right)\right\}$.

By integrating time from 0 to $T$ in (2.9) and taking limit as $n \rightarrow \infty$, we obtain

$$
\begin{aligned}
& (y(T), w(T))+a(y(T), w(T))-\int_{0}^{T}\left[\left(y, w_{t}^{\prime}\right)+a\left(y, w_{t}^{\prime}\right)+d(y, w)\right] \\
& \quad+\int_{0}^{T} \int_{0}^{t} c(t, \tau ; y(\tau), w(t)) d \tau d t \\
& =\left(y^{0}, w(0)\right)+a\left(y^{0}, w(0)\right)+\int_{0}^{T}(f+B u, w), \quad \forall w \in W .
\end{aligned}
$$

Then,

$$
\left(y_{t}, w\right)+a\left(y_{t}, w\right)+d(y, w)+\int_{0}^{t} c(t, \tau ; y(\tau), w) d \tau=(f+B u, w), \quad \forall w \in V, t \in(0, T]
$$

Furthermore, we have

$$
\int_{0}^{T}\left[\left(y_{t}, y_{t}\right)+a\left(y_{t}, y_{t}\right)+d\left(y, y_{t}\right)+\int_{0}^{t} c\left(t, \tau ; y(\tau), y_{t}(t)\right) d \tau\right] d t=\int_{0}^{T}\left(f+B u, y_{t}\right)
$$

Then, we get

$$
\int_{0}^{T}\left\|y_{t}\right\|_{1, \Omega}^{2} \leqslant C \int_{0}^{T}\left[\|f\|_{-1, \Omega}^{2}+\|u\|_{0, \Omega_{U}}^{2}+\|y\|_{1, \Omega}^{2}+\int_{0}^{t}\|y\|_{1, \Omega}^{2} d \tau\right] .
$$

This means $y_{t} \in L^{2}\left(0, T ; H^{1}(\Omega)\right)$. So $(u, y)$ is one solution of $(2.8)$.

Since $\int_{0}^{T}\left\|y-z_{d}\right\|_{0, \Omega}^{2}$ is a convex function on space $L^{2}\left(0, T ; L^{2}(\Omega)\right)$ and $(\alpha / 2) \int_{0}^{T}\|u\|_{0, \Omega_{U}}^{2}$ is a strictly convex function on $U$, hence $J(u, y(u))$ is a strictly convex function on $U$, so the minimization problem (2.8) has one unique solution. 


\subsection{Optimality Conditions and Their Finite Element Approximation}

By the theory of optimal control problem (see [18]), we can similarly deduce the following optimality conditions of the problem (2.8).

Theorem 2.2. A pair $(y, u) \in L^{2}\left(0, T ; H_{0}^{1}(\Omega)\right) \times L^{2}\left(0, T ; L^{2}\left(\Omega_{U}\right)\right)$ is the solution of the optimal control problem (2.8), if and only if there exists a costate $p \in L^{2}\left(0, T ; H_{0}^{1}(\Omega)\right)$ such that the triple $(y, p, u)$ satisfies the following optimality conditions:

$$
\begin{gathered}
\left(y_{t}, w\right)+a\left(y_{t}, w\right)+d(y, w)+\int_{0}^{t} c(t, \tau ; y(\tau), w(t)) d \tau=(f+B u, w) \\
\left.y\right|_{t=0}=y^{0} ; \quad \forall w \in V, t \in(0, T] \\
-\left(q, p_{t}\right)-a\left(q, p_{t}\right)+d(q, p)+\int_{t}^{T} c(\tau, t ; q(t), p(\tau)) d \tau=\left(y-z_{d}, q\right) \quad \forall q \in V, t \in[0, T), \\
\left.p\right|_{t=T}=0 ; \\
\int_{0}^{T}\left(u+B^{*} p, v-u\right)_{U} d t \geqslant 0, \quad \forall v \in U_{a d}
\end{gathered}
$$

where $B^{*}$ is the adjoint operator of $B$.

Let us consider the semi-discrete finite element approximation of the control problem (2.8). Here, we only consider triangular and conforming elements.

Let $\Omega^{h}$ be a polygonal approximation to $\Omega$ with boundary $\partial \Omega^{h}$. Let $T^{h}$ be a partitioning of $\Omega^{h}$ into disjoint regular $n$-simplices $\tau$, so that $\bar{\Omega}^{h}=\bigcup_{\tau \in T^{h}} \bar{\tau}$. Each element has at most one face on $\partial \Omega^{h}$, and $\bar{\tau}$ and $\bar{\tau}^{\prime}$ have either only one common vertex or a whole edge or face if $\bar{\tau}$ and $\bar{\tau}^{\prime} \in T^{h}$. We further require that $P_{i} \in \partial \Omega^{h} \Rightarrow P_{i} \in \partial \Omega$ where $P_{i} \quad(i=1, \ldots, J)$ is the vertex set associated with the triangulation $T_{h}$. As usual, $h$ denotes the diameter of the triangulation $T^{h}$. For simplicity, we assume that $\Omega$ is a convex polygon so that $\Omega=\Omega^{h}$.

Associated with $T^{h}$ is a finite-dimensional subspace $S^{h}$ of $C\left(\bar{\Omega}^{h}\right)$, such that $\left.\chi\right|_{\tau}$ are polynomials of order $m(m \geq 1)$ for all $x \in S^{h}$ and $\tau \in T^{h}$. Let $V^{h}=\left\{v_{h} \in S_{h}: \quad v_{h}\left(P_{i}\right)=0\right.$ $(i=1, \ldots, J)\}, W^{h}=L^{2}\left(0, T ; V^{h}\right)$. Note that we do not impose a continuity requirement. It is easy to see that $V^{h} \subset V, W^{h} \subset W$.

Let $T_{U}^{h}$ be a partitioning of $\Omega_{U}^{h}$ into disjoint regular $n$-simplices $\tau_{U}$, so that $\bar{\Omega}_{U}^{h}=$ $\bigcup_{\tau_{U} \in T_{U}^{h}} \bar{\tau}_{U} \cdot \bar{\tau}_{U}$ and $\bar{\tau}_{U}^{\prime}$ have either only one common vertex or a whole edge or face if $\bar{\tau}_{U}$ and $\bar{\tau}_{U}^{\prime} \in T_{U}^{h}$. We further require that $P_{i} \in \partial \Omega_{U}^{h} \Rightarrow P_{i} \in \partial \Omega_{U}$, where $P_{i} \quad(i=1, \ldots, J)$ is the vertex set associated with the triangulation $T_{U}^{h}$. For simplicity, we again assume that $\Omega_{U}$ is a convex polygon so that $\Omega_{U}=\Omega_{U}^{h}$.

Associated with $T_{U}^{h}$ is another finite-dimensional subspace $U^{h}$ of $L^{2}\left(\Omega_{U}^{h}\right)$, such that $\left.\chi\right|_{\tau_{U}}$ are polynomials of order $m(m \geqslant 0)$ for all $\chi \in U^{h}$ and $\tau_{U} \in T_{U}^{h}$. Here there is no requirement for the continuity. Let $X^{h}=L^{2}\left(0, T ; U^{h}\right)$. It is easy to see that $X^{h} \subset X$. Let $h_{\tau}\left(h_{\tau_{U}}\right)$ denote the maximum diameter of the element $\tau\left(\tau_{U}\right)$ in $T^{h}\left(T_{U}^{h}\right)$. 
Due to the limited regularity of the optimal control $u$ in general, there will be no advantage in considering higher-order finite element spaces than the piecewise constant space for the control. We therefore only consider the piecewise constant finite element space for the approximation of the control, though higher-order finite element spaces will be used to approximate the state and the co-state. Let $P_{0}(\Omega)$ denote all the 0 -order polynomial over $\Omega$. Therefore, we always take $X^{h}=\left\{u \in X:\left.u(x, t)\right|_{x \in \tau_{U}} \in P_{0}\left(\tau_{U}\right)\right.$, for all $\left.t \in[0, T]\right\} . U_{a d}^{h}$ is a closed convex set in $X^{h}$. For ease of exposition, in this paper, we assume that $U_{a d}^{h} \subset U_{a d} \cap X^{h}$. $(\mathrm{OCP})^{h}$ :

Then a possible semi-discrete finite element approximation of (OCP) is as follows

$$
\min _{u_{h} \in U_{a d}^{h}} J\left(u_{h}, y_{h}\right)=\frac{1}{2}\left\{\int_{0}^{T}\left\|y_{h}-z_{d}\right\|_{0, \Omega}^{2}+\int_{0}^{T}\left\|u_{h}\right\|_{0, \Omega_{U}}^{2}\right\},
$$

such that

$$
\begin{aligned}
& \left(\frac{\partial y_{h}}{\partial t}, w_{h}\right)+a\left(\frac{\partial y_{h}}{\partial t}, w_{h}\right)+d\left(y_{h}, w_{h}\right)+\int_{0}^{t} c\left(t, \tau ; y_{h}(\tau), w_{h}(t)\right) d \tau=\left(f+B u_{h}, w_{h}\right) \\
& \forall w_{h} \in V^{h}, t \in(0, T] \\
& \left.y_{h}\right|_{t=0}=y_{h^{\prime}}^{0}
\end{aligned}
$$

where $y_{h} \in W^{h}$ and $y_{h}^{0} \in V^{h}$ is the approximation of $y^{0}$.

In the same way of proving Theorem 2.1, we can easily prove that the problem (2.20)(2.21) has a unique solution $\left(y_{h}, u_{h}\right) \in W^{h} \times U_{a d}^{h}$.

It is well known (see [18]) that a pair $\left(y_{h}, u_{h}\right) \in W^{h} \times U_{a d}^{h}$ is a solution of (2.20)(2.21), if and only if there exists a co-state $p_{h} \in W^{h}$ such that the triple $\left(y_{h}, p_{h}, u_{h}\right)$ satisfies the following optimality conditions:

$$
\begin{gathered}
\left(\frac{\partial y_{h}}{\partial t}, w_{h}\right)+a\left(\frac{\partial y_{h}}{\partial t}, w_{h}\right)+d\left(y_{h}, w_{h}\right)+\int_{0}^{t} c\left(t, \tau ; y_{h}(\tau), w_{h}(t)\right) d \tau=\left(f+B u_{h}, w_{h}\right), \\
\left.y_{h}\right|_{t=0}=y_{h^{\prime}}^{0} \\
-\left(q_{h}, \frac{\partial p_{h}}{\partial t}\right)-a\left(w_{h} \in V^{h},\right. \\
\left.p_{h}\right|_{t=T}=0, \\
\int_{0}^{T}\left(u_{h}+B^{*} p_{h}, v_{h}-u_{h}\right)_{U} d t \geqslant 0, \quad \forall v_{h} \in U_{a d}^{h} .
\end{gathered}
$$


The optimality conditions in (2.22)-(2.24) are the semi-discrete approximation to the problem (2.17)-(2.19).

Introduce the local averaging operator $\pi_{h}$ given by

$$
\left.\left(\pi_{h} w\right)\right|_{\tau_{U}}:=\frac{\int_{\tau_{U}} w}{\int_{\tau_{U}} 1}, \quad \forall \tau_{U} \in T_{U}^{h} .
$$

Then, we have $\int_{\Omega_{U}} w=\int_{\Omega_{U}} \pi_{h} w$ for any $w \in L^{2}\left(0, T ; L^{2}\left(\Omega_{U}\right)\right), t \in[0, T]$ and (2.24) is equivalent to

$$
\int_{0}^{T}\left(u_{h}+\pi_{h}\left(B^{*} p_{h}\right), v_{h}-u_{h}\right)_{U} d t \geqslant 0, \quad \forall v_{h} \in U_{a d}^{h}
$$

In the following, we derive the a posteriori error estimates for semi-discrete finite element approximation (2.22)-(2.24), allowing different meshes to be used for the state and the control.

The following lemmas are important in deriving the a posteriori error estimates of residual type.

Lemma 2.3 (see [19]). Let $\widehat{\pi}_{h}$ be the standard Lagrange interpolation operator. For $m=0$ or 1 , $q>n / 2$ and $v \in W^{2, q}(\Omega)$ as

$$
\left|v-\widehat{\pi}_{h} v\right|_{m, q, \Omega} \leqslant C h^{2-m}|v|_{2, q, \Omega}
$$

Lemma 2.4 (see [20]). Let $\pi_{h}$ be the average interpolation operator defined in (2.25). For $m=0$ or $1,1 \leqslant q \leqslant \infty$ and for all $v \in W^{1, q}\left(\Omega^{h}\right)$ as

$$
\left|v-\pi_{h} v\right|_{m, q, \tau} \leqslant \sum_{\bar{\tau}^{\prime} \cap \bar{\tau} \neq \emptyset} C h_{\tau}^{1-m}|v|_{1, q, \tau^{\prime}}
$$

Lemma 2.5 (see [21]). For all $v \in W^{1, q}(\Omega), 1 \leqslant q<\infty$ as

$$
\|v\|_{0, q, \partial \tau} \leqslant C\left(h_{\tau}^{-1 / q}\|v\|_{0, q, \tau}+h_{\tau}^{1-1 / q}|v|_{1, q, \tau}\right) .
$$

\section{A Posteriori Error Estimates in $L^{\infty}\left(0, T ; H^{1}(\Omega)\right)$-Norm}

In this paper, the control constraints are given in an integral sense as follows:

$$
U_{a d}=\left\{v \in X ; \int_{\Omega_{U}} v \geqslant 0, t \in[0, T]\right\}
$$

The following lemma is the first step to derive the a posteriori error estimates of residual type. 
Lemma 3.1. Let $(y, p, u)$ and $\left(y_{h}, p_{h}, u_{h}\right)$ be the solutions of (2.17)-(2.19) and (2.22)-(2.24). Then, we have

$$
\left\|u-u_{h}\right\|_{L^{2}\left(0, T ; L^{2}\left(\Omega_{U}\right)\right)}^{2} \leqslant C \eta_{1}^{2}+C\left\|p_{h}-p\left(u_{h}\right)\right\|_{L^{2}\left(0, T ; L^{2}(\Omega)\right)^{\prime}}^{2}
$$

where

$$
\eta_{1}^{2}=\int_{0}^{T}\left\{\sum_{\tau_{U}} \int_{\tau_{U}}\left(B^{*} p_{h}-P_{h}\left(B^{*} p_{h}\right)\right)^{2}\right\} d t
$$

$P_{h}$ is the $L^{2}$-projection from $L^{2}(\Omega)$ to $U^{h}$, and $p\left(u_{h}\right)$ is defined by the following system:

$$
\begin{gathered}
\left(\frac{\partial}{\partial t} y\left(u_{h}\right), \omega\right)+a\left(\frac{\partial}{\partial t} y\left(u_{h}\right), \omega\right)+d\left(y\left(u_{h}\right), \omega\right)+\int_{0}^{t} c\left(t, \tau ; y\left(u_{h}\right)(\tau), \omega(t)\right) d \tau \\
=\left(f+B u_{h}, \omega\right), \quad \forall \omega \in V, \\
y\left(u_{h}\right)(0)=y_{0}^{h}(x), \quad x \in \Omega, \\
-\left(q, \frac{\partial}{\partial t} p\left(u_{h}\right)\right)-a\left(q, \frac{\partial}{\partial t} p\left(u_{h}\right)\right)+d\left(q, p\left(u_{h}\right)\right)+\int_{t}^{T} c\left(\tau, t ; q(t), p\left(u_{h}\right)(\tau)\right) d \tau \\
=\left(y\left(u_{h}\right)-z_{d}, q\right), \quad \forall q \in V .
\end{gathered}
$$

Proof. From (2.19), we have

$$
\left(u, u-u_{h}\right)_{U} \leqslant-\left(B^{*} p, u-u_{h}\right)_{U}
$$

Then, by (2.24) and (3.6), we have

$$
\begin{aligned}
\left\|u-u_{h}\right\|_{L^{2}\left(0, T ; L^{2}\left(\Omega_{U}\right)\right)}^{2}= & \int_{0}^{T}\left[\left(u, u-u_{h}\right)_{U}-\left(u_{h}, u-u_{h}\right)_{U}\right] d t=\int_{0}^{T}-\left(B^{*} p+u_{h}, u-u_{h}\right)_{U} d t \\
= & -\int_{0}^{T}\left(B^{*} p_{h}+u_{h}, u-v_{h}\right)_{U} d t-\int_{0}^{T}\left(B^{*} p_{h}+u_{h}, v_{h}-u_{h}\right)_{U} d t \\
& +\int_{0}^{T}\left(B^{*} p_{h}-B^{*} p\left(u_{h}\right), u-u_{h}\right)_{U} d t+\int_{0}^{T}\left(B^{*} p\left(u_{h}\right)-B^{*} p, u-u_{h}\right)_{U} d t \\
\leqslant & \inf _{v_{h} \in U_{a d}^{h}} \int_{0}^{T}\left(B^{*} p_{h}+u_{h}, v_{h}-u\right)_{U} d t \\
& +\int_{0}^{T}\left(B^{*}\left(p_{h}-p\left(u_{h}\right)\right), u-u_{h}\right)_{U} d t+\int_{0}^{T}\left(B^{*}\left(p\left(u_{h}\right)-p\right), u-u_{h}\right)_{U} d t \\
= & I_{1}+I_{2}+I_{3} .
\end{aligned}
$$

Next, we will estimate $I_{1}, I_{2}$, and $I_{3}$, respectively. 
(1) We first estimate $I_{1}$. Let $P_{h}$ be the $L^{2}$-projection from $L^{2}(\Omega)$ to $U^{h}$.

We have

$$
\int_{\Omega_{U}}\left(P_{h} v-v\right) \phi=0, \quad \forall \phi \in X^{h}, v \in U_{a d}, t \in(0, T]
$$

Since $v \in U_{a d}$, so $\int_{\Omega_{u}} P_{h} v \geqslant 0$, then $P_{h} v \in U_{a d}^{h}$. So that we can take $v_{h}=P_{h} u$ in $I_{1}$.

For given $t \in(0, T]$, let

$$
u_{h}=P_{h}\left(-B^{*} p_{h}+\max \left\{0, \frac{\int_{\Omega_{U}} B^{*} p_{h}}{\int_{\Omega_{U}} 1}\right\}\right)
$$

We have $u_{h} \in X^{h}$. We will show that $u_{h}$ is the solution of the variational inequality in (2.24) assuming $p_{h}$ is known. we have

Since $\int_{\Omega_{U}}\left[P_{h}\left(-B^{*} p_{h}+\max \left\{0, \int_{\Omega_{U}} B^{*} p_{h} / \int_{\Omega_{U}} 1\right\}\right)-\left(-B^{*} p_{h}+\max \left\{0, \int_{\Omega_{U}} B^{*} p_{h} / \int_{\Omega_{U}} 1\right\}\right)\right]=0$,

$$
\int_{\Omega_{U}} u_{h}=-\int_{\Omega_{U}} B^{*} p_{h}+\int_{\Omega_{U}} \max \left\{0, \frac{\int_{\Omega_{U}} B^{*} p_{h}}{\int_{\Omega_{U}} 1}\right\}= \begin{cases}-\int_{\Omega_{U}} B^{*} p_{h}, & \int_{\Omega_{U}} B^{*} p_{h}<0 \\ 0, & \int_{\Omega_{U}} B^{*} p_{h} \geqslant 0\end{cases}
$$

Thus, $\int_{\Omega_{U}} u_{h} \geqslant 0$, we have $u_{h} \in U_{a d}^{h}$. Note that for all $v_{h} \in U_{a d}^{h}, t \in(0, T]$, we have

$$
\begin{aligned}
& \left(u_{h}+B^{*} p_{h}, v_{h}-u_{h}\right)_{U} \\
& =\int_{\Omega_{U}}\left[P_{h}\left(-B^{*} p_{h}+\max \left\{0, \frac{\int_{\Omega_{U}} B^{*} p_{h}}{\int_{\Omega_{U}} 1}\right\}\right)\right. \\
& \left.\quad-\left(-B^{*} p_{h}+\max \left\{0, \frac{\int_{\Omega_{U}} B^{*} p_{h}}{\int_{\Omega_{U}} 1}\right\}\right)+\max \left\{0, \frac{\int_{\Omega_{U}} B^{*} p_{h}}{\int_{\Omega_{U}} 1}\right\}\right]\left(v_{h}-u_{h}\right) \\
& =\int_{\Omega_{U}} \max \left\{0, \frac{\int_{\Omega_{U}} B^{*} p_{h}}{\int_{\Omega_{U}} 1}\right\}\left(v_{h}-u_{h}\right) .
\end{aligned}
$$

If $\int_{\Omega_{U}} B^{*} p_{h}<0$, then

$$
\left(u_{h}+B^{*} p_{h}, v_{h}-u_{h}\right)_{U}=\int_{\Omega_{U}} 0 \cdot\left(v_{h}-u_{h}\right)=0 .
$$

If $\int_{\Omega_{U}} B^{*} p_{h} \geqslant 0$, since

$$
\int_{\Omega_{U}} u_{h}=\int_{\Omega_{U}}\left(-B^{*} p_{h}+\max \left\{0, \frac{\int_{\Omega_{U}} B^{*} p_{h}}{\int_{\Omega_{U}} 1}\right\}\right)=0
$$


we have

$$
\left(u_{h}+B^{*} p_{h}, v_{h}-u_{h}\right)_{U}=\frac{\int_{\Omega_{U}} B^{*} p_{h}}{\int_{\Omega_{U}} 1} \int_{\Omega_{U}}\left(v_{h}-u_{h}\right)=\frac{\int_{\Omega_{U}} B^{*} p_{h}}{\int_{\Omega_{U}} 1} \cdot \int_{\Omega_{U}} v_{h} \geqslant 0 .
$$

From (3.11)-(3.14), we obtain

$$
\left(u_{h}+B^{*} p_{h}, v_{h}-u_{h}\right)_{U} \geqslant 0, \quad \forall v_{h} \in U_{a d}^{h}
$$

So $u_{h}=P_{h}\left(-B^{*} p_{h}+\max \left\{0, \int_{\Omega_{U}} B^{*} p_{h} / \int_{\Omega_{U}} 1\right\}\right)$ is the solution of the variational inequality in (2.24) assuming $p_{h}$ is known.

Then,

$$
\begin{aligned}
I_{1} & \leqslant \int_{0}^{T}\left(B^{*} p_{h}+u_{h}, P_{h} u-u\right)_{U} d t \\
& =\int_{0}^{T}\left\{\sum_{\tau_{U}} \int_{\tau_{u}}\left[P_{h}\left(-B^{*} p_{h}+\max \left\{0, \frac{\int_{\Omega_{U}} B^{*} p_{h}}{\int_{\Omega_{U}} 1}\right\}\right)+B^{*} p_{h}\right]\left(P_{h} u-u\right)\right\} d t .
\end{aligned}
$$

Since $\int_{\tau_{u}}\left(P_{h} u-u\right)=0$, we have

$$
\begin{aligned}
I_{1} & \leqslant \int_{0}^{T}\left\{\sum_{\tau_{U}} \int_{\tau_{U}}\left(-P_{h}\left(B^{*} p_{h}\right)+B^{*} p_{h}\right)\left(P_{h} u-u\right)\right\} d t \\
& =\int_{0}^{T}\left\{\sum_{\tau_{U}} \int_{\tau_{U}}\left(-P_{h}\left(B^{*} p_{h}\right)+B^{*} p_{h}\right)\left(P_{h}\left(u-u_{h}\right)-\left(u-u_{h}\right)\right)\right\} d t \\
& \leqslant C(\delta) \int_{0}^{T}\left\{\sum_{\tau_{U}} \int_{\tau_{U}}\left(-P_{h}\left(B^{*} p_{h}\right)+B^{*} p_{h}\right)^{2}\right\} d t+\delta\left\|u-u_{h}\right\|_{L^{2}\left(0, T ; L^{2}\left(\Omega_{U}\right)\right)}^{2} \\
& =C \eta_{1}^{2}+\delta\left\|u-u_{h}\right\|_{L^{2}\left(0, T ; L^{2}\left(\Omega_{U}\right)\right)^{2}}^{2}
\end{aligned}
$$

(2) Consider

$$
I_{2}=\int_{0}^{T}\left(B^{*}\left(p_{h}-p\left(u_{h}\right)\right), u-u_{h}\right)_{U} d t \leqslant C\left\|p_{h}-p\left(u_{h}\right)\right\|_{L^{2}\left(0, T ; L^{2}(\Omega)\right)}^{2}+\delta\left\|u-u_{h}\right\|_{L^{2}\left(0, T ; L^{2}\left(\Omega_{U}\right)\right)}^{2} .
$$

(3) By (3.4) and (2.17), we have for $t \in(0, T]$

$$
\begin{aligned}
\left(\frac{\partial}{\partial t}\left(y-y\left(u_{h}\right)\right), \omega\right)+a\left(\frac{\partial}{\partial t}\left(y-y\left(u_{h}\right)\right), \omega\right)+d\left(y-y\left(u_{h}\right), w\right) \\
\quad+\int_{0}^{t} c\left(t, \tau ;\left(y-y\left(u_{h}\right)\right)(\tau), \omega(t)\right) d \tau=\left(B\left(u-u_{h}\right), \omega\right), \quad \forall \omega \in V
\end{aligned}
$$


and from (3.5) and (2.18), we have

$$
\begin{aligned}
&-(q,\left.\frac{\partial}{\partial t}\left(p-p\left(u_{h}\right)\right)\right)-a\left(q, \frac{\partial}{\partial t}\left(p-p\left(u_{h}\right)\right)\right)+d\left(q, p-p\left(u_{h}\right)\right) \\
&+\int_{t}^{T} c\left(\tau, t ; q(t),\left(p-p\left(u_{h}\right)\right)(\tau)\right) d \tau=\left(y-y\left(u_{h}\right), q\right), \quad \forall q \in V .
\end{aligned}
$$

Then, from (3.19), (3.20), and integrating by part we have

$$
\begin{aligned}
I_{3}=\int_{0}^{T}\left(B^{*}\left(p\left(u_{h}\right)-p\right), u-u_{h}\right)_{U} d t=\int_{0}^{T}\left(p\left(u_{h}\right)-p, B\left(u-u_{h}\right)\right)_{U} d t \\
=\int_{0}^{T}\left[\left(\frac{\partial}{\partial t}\left(y-y\left(u_{h}\right)\right), p\left(u_{h}\right)-p\right)+a\left(\frac{\partial}{\partial t}\left(y-y\left(u_{h}\right)\right), p\left(u_{h}\right)-p\right)\right. \\
\left.\quad+d\left(y-y\left(u_{h}\right), p\left(u_{h}\right)-p\right)+\int_{0}^{t} c\left(t, \tau ;\left(y-y\left(u_{h}\right)\right)(\tau),\left(p\left(u_{h}\right)-p\right)(t)\right) d \tau\right] d t \\
=\int_{0}^{T}\left[-\left(y-y\left(u_{h}\right), \frac{\partial}{\partial t}\left(p\left(u_{h}\right)-p\right)\right)-a\left(y-y\left(u_{h}\right), \frac{\partial}{\partial t}\left(p\left(u_{h}\right)-p\right)\right)\right. \\
\left.\quad+d\left(y-y\left(u_{h}\right), p\left(u_{h}\right)-p\right)+\int_{t}^{T} c\left(\tau, t ;\left(y-y\left(u_{h}\right)\right)(t),\left(p\left(u_{h}\right)-p\right)(\tau)\right) d \tau\right] d t \\
=\int_{0}^{T}-\left(y-y\left(u_{h}\right), y-y\left(u_{h}\right)\right) d t \leqslant 0 .
\end{aligned}
$$

Following from (3.17)-(3.21), let $\delta$ be small enough as

$$
\left\|u-u_{h}\right\|_{L^{2}\left(0, T ; L^{2}\left(\Omega_{u}\right)\right)}^{2} \leqslant C \eta_{1}^{2}+C\left\|p_{h}-p\left(u_{h}\right)\right\|_{L^{2}\left(0, T ; L^{2}(\Omega)\right)}^{2} .
$$

This completes the proof.

Lemma 3.2. Let $(y, p, u)$ and $\left(y_{h}, p_{h}, u_{h}\right)$ be the solutions of $(2.17)-(2.19)$, and (2.22)-(2.24) respectively. Then, there hold the a posteriori error estimates as

$$
\begin{aligned}
& \left\|y_{h}-y\left(u_{h}\right)\right\|_{L^{\infty}\left(0, T ; H^{1}(\Omega)\right)}^{2}+\left\|\frac{\partial}{\partial t}\left(y_{h}-y\left(u_{h}\right)\right)\right\|_{L^{2}\left(0, T ; H^{1}(\Omega)\right)}^{2} \\
& +\left\|p_{h}-p\left(u_{h}\right)\right\|_{L^{\infty}\left(0, T ; H^{1}(\Omega)\right)}^{2}+\left\|\frac{\partial}{\partial t}\left(p_{h}-p\left(u_{h}\right)\right)\right\|_{L^{2}\left(0, T ; H^{1}(\Omega)\right)}^{2} \leqslant C \sum_{i=2}^{6} \eta_{i}^{2},
\end{aligned}
$$


where

$$
\begin{aligned}
& \eta_{2}^{2}=\int_{0}^{T}\left\{\sum _ { \tau } h _ { \tau } ^ { 2 } \int _ { \tau } \left(\frac{\partial p_{h}}{\partial t}-\operatorname{div}\left(A^{*} \nabla \frac{\partial p_{h}}{\partial t}\right)+\operatorname{div}\left(D^{*} \nabla p_{h}\right)\right.\right. \\
& \left.\left.\quad+\int_{t}^{T} \operatorname{div}\left(C^{*}(\tau, t) \nabla p_{h}(\tau)\right) d \tau+y_{h}-z_{d}\right)^{2} d \tau\right\} d t, \\
& \eta_{3}^{2}=\int_{0}^{T} \sum_{\tau} h_{l} \int_{\partial \tau}\left[-\left(A^{*} \nabla \frac{\partial p_{h}}{\partial t}\right) \cdot n+\left(D^{*} \nabla p_{h}\right) \cdot n+\int_{t}^{T}\left(C^{*}(\tau, t) \nabla p_{h}(\tau)\right) \cdot n d \tau\right]^{2} d l d t, \\
& \eta_{4}^{2}=\int_{0}^{T}\left\{\sum _ { \tau } h _ { \tau } ^ { 2 } \int _ { \tau } \left(\frac{\partial}{\partial t} y_{h}-\operatorname{div}\left(A \nabla \frac{\partial y_{h}}{\partial t}\right)-\operatorname{div}\left(D \nabla y_{h}\right)\right.\right. \\
& \left.\left.\quad-\int_{0}^{t} \operatorname{div}\left(C(t, \tau) \nabla y_{h}(\tau)\right) d \tau-f-B u_{h}\right)^{2}\right\} d t, \\
& \eta_{5}^{2}=\int_{0}^{T} \sum_{\tau} h_{l} \int_{\partial \tau}\left[\left(A \nabla \frac{\partial y_{h}}{\partial t}\right) \cdot n+\left(A \nabla y_{h}\right) \cdot n+\int_{0}^{t}\left(C(t, \tau) \nabla y_{h}(\tau)\right) \cdot n d \tau\right]^{2} d l d t, \\
& \eta_{6}^{2}=\left\|y_{0}^{h}-y_{0}\right\|_{1, \Omega^{\prime}}^{2}
\end{aligned}
$$

where $l$ is a face of an element $\tau, h_{l}$ is the maximum diameter of $l$, and $\left[\nabla p_{h} \cdot n\right]$ and $\left[\nabla y_{h} \cdot n\right]$ are the normal derivative jumps over the interior face $l$ defined by

$$
\begin{gathered}
{\left[\nabla p_{h} \cdot n\right]_{l}=\left(\left.\nabla p_{h} h\right|_{\tau_{l}^{1}}-\left.\nabla p_{h}\right|_{\tau_{l}^{2}}\right) \cdot n,} \\
{\left[\nabla y_{h} \cdot n\right]_{l}=\left(\left.\nabla y_{h}\right|_{\tau_{l}^{1}}-\left.\nabla y_{h}\right|_{\tau_{l}^{2}}\right) \cdot n,}
\end{gathered}
$$

where $n$ is the unit normal vector on $l=\tau_{l}^{1} \cap \tau_{l}^{2}$ outwards $\tau_{l}^{1}$. For later convenience, one can define $\left[\nabla p_{h} \cdot n\right]_{l}=0$ and $\left[\nabla y_{h} \cdot n\right]_{l}=0$ when $l \subset \partial \Omega$.

Proof. Let

$$
\begin{aligned}
\left\langle R\left(u_{h}\right), v\right\rangle= & -\left(v, \frac{\partial}{\partial t}\left(p_{h}-p\left(u_{h}\right)\right)\right)-a\left(v, \frac{\partial}{\partial t}\left(p_{h}-p\left(u_{h}\right)\right)\right)+d\left(v, p_{h}-p\left(u_{h}\right)\right) \\
& +\int_{t}^{T} c\left(\tau, t ; v(t),\left(p_{h}-p\left(u_{h}\right)\right)(\tau)\right) d \tau,
\end{aligned}
$$

and $\pi_{h}$ the average interpolation operator defined as in (2.25) and $e=p_{h}-p\left(u_{h}\right)$. Then, it follows from (2.23) and (3.5) that

$$
\begin{aligned}
& -\left(q_{h}, \frac{\partial}{\partial t}\left(p_{h}-p\left(u_{h}\right)\right)\right)-a\left(q_{h}, \frac{\partial}{\partial t}\left(p_{h}-p\left(u_{h}\right)\right)\right)+d\left(q_{h}, p_{h}-p\left(u_{h}\right)\right) \\
& \quad+\int_{t}^{T} c\left(\tau, t ; q_{h}(t),\left(p_{h}-p\left(u_{h}\right)\right)(\tau)\right) d \tau=\left(y_{h}-y\left(u_{h}\right), q_{h}\right), \quad \forall q_{h} \in V^{h} .
\end{aligned}
$$


We have

$$
\begin{aligned}
\langle & \left.R\left(u_{h}\right), v\right\rangle \\
= & -\left(v-\pi_{h} v, \frac{\partial}{\partial t}\left(p_{h}-p\left(u_{h}\right)\right)\right)-a\left(v-\pi_{h} v, \frac{\partial}{\partial t}\left(p_{h}-p\left(u_{h}\right)\right)\right)+d\left(v-\pi_{h} v, p_{h}-p\left(u_{h}\right)\right) \\
& +\int_{t}^{T} c\left(\tau, t ;\left(v-\pi_{h} v\right)(t),\left(p_{h}-p\left(u_{h}\right)\right)(\tau)\right) d \tau-\left(\pi_{h} v, \frac{\partial}{\partial t}\left(p_{h}-p\left(u_{h}\right)\right)\right) \\
& -a\left(\pi_{h} v, \frac{\partial}{\partial t}\left(p_{h}-p\left(u_{h}\right)\right)\right)+d\left(\pi_{h} v, p_{h}-p\left(u_{h}\right)\right)+\int_{t}^{T} c\left(\tau, t ; \pi_{h} v(t),\left(p_{h}-p\left(u_{h}\right)\right)(\tau)\right) d \tau \\
= & -\left(v-\pi_{h} v, \frac{\partial p_{h}}{\partial t}\right)-a\left(v-\pi_{h} v, \frac{\partial p_{h}}{\partial t}\right)+d\left(v-\pi_{h} v, p_{h}\right)+\int_{t}^{T} c\left(\tau, t ;\left(v-\pi_{h} v\right)(t), p_{h}(\tau)\right) d \tau \\
& -\left(y\left(u_{h}\right)-z_{d}, v-\pi_{h} v\right)+\left(y_{h}-y\left(u_{h}\right), \pi_{h} v\right) \\
= & \sum_{\tau} \int_{\tau}\left(-\frac{\partial p_{h}}{\partial t}+\operatorname{div}\left(A^{*} \nabla \frac{\partial p_{h}}{\partial t}\right)-\operatorname{div}\left(D^{*} \nabla p_{h}\right)-\int_{t}^{T} \operatorname{div}\left(C^{*}(\tau, t) \nabla p_{h}(\tau)\right) d \tau-y_{h}+z_{d}\right) \\
& \times\left(v-\pi_{h} v\right)+\sum_{\tau} \int_{\partial \tau}\left[-\left(A^{*} \nabla \frac{\partial p_{h}}{\partial t}\right) \cdot n+\left(D^{*} \nabla p_{h}\right) \cdot n+\int_{t}^{T}\left(C^{*}(\tau, t) \nabla p_{h}(\tau)\right) \cdot n d \tau\right] \\
& \times\left(v-\pi_{h} v\right)+\left(y_{h}-y\left(u_{h}\right), v\right) \\
\leqslant & \left\{\sum _ { \tau } \int _ { \tau } h _ { \tau } ^ { 2 } \left(-\frac{\partial p_{h}}{\partial t}+\operatorname{div}\left(A^{*} \nabla \frac{\partial p_{h}}{\partial t}\right)-\operatorname{div}\left(D^{*} \nabla p_{h}\right)\right.\right. \\
& \left.\left.\left.+\|_{t}^{T} \operatorname{div}\left(\|_{1, \Omega}^{*}(\tau, t) \nabla p_{h}(\tau)\right) d \tau-y_{h}+z_{d}\right)^{2} h_{h}\left[-\left(A^{*} \nabla \frac{\partial p_{h}}{\partial t}\right) \cdot n+\left(D^{*} \nabla p_{h}\right) \cdot n+\int_{t}^{T}\left(C^{*}(\tau, t) \nabla p_{h}(\tau)\right) \cdot n d \tau\right]^{2}\right\}^{1 / 2}, v\right) \\
& +(3,28)
\end{aligned}
$$

Taking $v=p_{h}-p\left(u_{h}\right)$ in (3.28) and from (2.6), we have

$$
\begin{gathered}
-\frac{1}{2} \frac{d}{d t}\left\|p_{h}-p\left(u_{h}\right)\right\|_{0, \Omega}^{2}-\frac{1}{2} \frac{d}{d t} a\left(p_{h}-p\left(u_{h}\right), p_{h}-p\left(u_{h}\right)\right)+c\left\|p_{h}-p\left(u_{h}\right)\right\|_{1, \Omega}^{2} \\
\leqslant\left\{\sum _ { \tau } \int _ { \tau } h _ { \tau } ^ { 2 } \left(-\frac{\partial p_{h}}{\partial t}+\operatorname{div}\left(A^{*} \nabla \frac{\partial p_{h}}{\partial t}\right)-\operatorname{div}\left(D^{*} \nabla p_{h}\right)-\int_{t}^{T} \operatorname{div}\left(C^{*}(\tau, t) \nabla p_{h}(\tau)\right) d \tau\right.\right. \\
\left.\left.-y_{h}+z_{d}\right)^{2}+\sum_{\tau} \int_{\partial \tau} h_{l}\left[-\left(A^{*} \nabla \frac{\partial p_{h}}{\partial t}\right) \cdot n+\left(D^{*} \nabla p_{h}\right) \cdot n\right]^{2}\right\}^{1 / 2}
\end{gathered}
$$


Abstract and Applied Analysis

$$
\begin{aligned}
& \times\left\|p_{h}-p\left(u_{h}\right)\right\|_{1, \Omega}+\left(y_{h}-y\left(u_{h}\right), p_{h}-p\left(u_{h}\right)\right) \\
& \quad-\int_{t}^{T} c\left(\tau, t ;\left(p_{h}-p\left(u_{h}\right)\right)(t),\left(p_{h}-p\left(u_{h}\right)\right)(\tau)\right) d \tau .
\end{aligned}
$$

Integrating time from $t$ to $T$ in (3.29) and by Schwartz inequality, Lemmas 2.4 and 2.5, we have

$$
\begin{aligned}
& \frac{1}{2}\left\|p_{h}-p\left(u_{h}\right)\right\|_{0, \Omega}^{2}+c\left\|p_{h}-p\left(u_{h}\right)\right\|_{1, \Omega}^{2}+c \int_{t}^{T}\left\|p_{h}-p\left(u_{h}\right)\right\|_{1, \Omega}^{2} d \tau \\
& \leqslant \int_{t}^{T} \sum_{\tau} h_{\tau}^{2} \times \int_{\tau}\left(\frac{\partial p_{h}}{\partial t}-\operatorname{div}\left(A^{*} \nabla \frac{\partial p_{h}}{\partial t}\right)+\operatorname{div}\left(D^{*} \nabla p_{h}\right)\right. \\
& \left.+\int_{\tau}^{T} \operatorname{div}\left(C^{*}(s, \tau) \nabla p_{h}(s)\right) d s+y_{h}-z_{d}\right)^{2} d \tau \\
& +\int_{t}^{T} \sum_{\tau} h_{l} \int_{\partial \tau}\left[-\left(A^{*} \nabla \frac{\partial p_{h}}{\partial t}\right) \cdot n+\left(D^{*} \nabla p_{h}\right) \cdot n+\int_{\tau}^{T}\left(C^{*}(s, \tau) \nabla p_{h}(s)\right) \cdot n d s\right]^{2} d \tau \\
& +\delta \int_{t}^{T}\left\|p_{h}-p\left(u_{h}\right)\right\|_{1, \Omega}^{2} d \tau+C \int_{t}^{T}\left\|y_{h}-y\left(u_{h}\right)\right\|_{0, \Omega}^{2} d \tau+C \int_{t}^{T} \int_{\tau}^{T}\left\|\left(p_{h}-p\left(u_{h}\right)\right)(s)\right\|_{1, \Omega}^{2} d s d \tau .
\end{aligned}
$$

Letting $\delta$ be small enough, we have

$$
\begin{gathered}
\int_{t}^{T}\left\|p_{h}-p\left(u_{h}\right)\right\|_{1, \Omega}^{2} d \tau \\
\leqslant C \int_{t}^{T} \sum_{\tau} h_{\tau}^{2} \int_{\tau}\left(\frac{\partial p_{h}}{\partial t}-\operatorname{div}\left(A^{*} \nabla \frac{\partial p_{h}}{\partial t}\right)+\operatorname{div}\left(D^{*} \nabla p_{h}\right)\right. \\
\left.+\int_{\tau}^{T} \operatorname{div}\left(C^{*}(s, \tau) \nabla p_{h}(s)\right) d s+y_{h}-z_{d}\right) d \tau \\
+C \int_{t}^{T} \sum_{\tau} h_{l} \int_{\partial \tau}\left[-\left(A^{*} \nabla \frac{\partial p_{h}}{\partial t}\right) \cdot n+\left(D^{*} \nabla p_{h}\right) \cdot n\right. \\
\left.+\int_{\tau}^{T}\left(C^{*}(s, \tau) \nabla p_{h}(s)\right) \cdot n d s\right]^{2} d \tau \\
+C \int_{t}^{T}\left\|y_{h}-y\left(u_{h}\right)\right\|_{0, \Omega}^{2} d \tau+C \int_{t}^{T} \int_{\tau}^{T}\left\|\left(p_{h}-p\left(u_{h}\right)\right)(s)\right\|_{1, \Omega}^{2} d s d \tau .
\end{gathered}
$$

Then, from Gronwall inequality and (3.28)-(3.31) we have

$$
\left\|p_{h}-p\left(u_{h}\right)\right\|_{L^{2}\left(0, T ; H^{1}(\Omega)\right)}^{2} \leqslant C \eta_{2}^{2}+C \eta_{3}^{2}+C\left\|y_{h}-y\left(u_{h}\right)\right\|_{L^{2}\left(0, T ; L^{2}(\Omega)\right)}^{2} .
$$


Similarly,

$$
\begin{aligned}
\left\|p_{h}-p\left(u_{h}\right)\right\|_{L^{\infty}\left(0, T ; H^{1}(\Omega)\right)}^{2} \leqslant & C\left(\eta_{2}^{2}+\eta_{3}^{2}+\left\|y_{h}-y\left(u_{h}\right)\right\|_{L^{2}\left(0, T ; L^{2}(\Omega)\right)}^{2}\right) \\
& +C \int_{0}^{T} \int_{t}^{T}\left\|\left(p_{h}-p\left(u_{h}\right)\right)(\tau)\right\|_{1, \Omega}^{2} d \tau d t \\
\leqslant & C \eta_{2}^{2}+C \eta_{3}^{2}+C\left\|y_{h}-y\left(u_{h}\right)\right\|_{L^{2}\left(0, T ; L^{2}(\Omega)\right)}^{2} .
\end{aligned}
$$

In the same way of getting (3.32), by setting $v=(\partial / \partial t)\left(p_{h}-p\left(u_{h}\right)\right)$ in $(3.28)$, we have

$$
\left\|\frac{\partial}{\partial t}\left(p_{h}-p\left(u_{h}\right)\right)\right\|_{L^{2}\left(0, T ; H^{1}(\Omega)\right)}^{2} \leqslant C \eta_{2}^{2}+C \eta_{3}^{2}+C\left\|y_{h}-y\left(u_{h}\right)\right\|_{L^{2}\left(0, T ; L^{2}(\Omega)\right)}^{2} .
$$

Similarly analysis for $\left\|y_{h}-y\left(u_{h}\right)\right\|_{L^{\infty}\left(0, T ; H^{1}(\Omega)\right)}$, we let

$$
\begin{aligned}
\left\langle Q\left(u_{h}\right), v\right\rangle= & \left(\frac{\partial}{\partial_{t}}\left(y_{h}-y\left(u_{h}\right)\right), v\right)+a\left(\frac{\partial}{\partial_{t}}\left(y_{h}-y\left(u_{h}\right)\right), v\right)+d\left(y_{h}-y\left(u_{h}\right), v\right) \\
& +\int_{0}^{t} c\left(t, \tau ;\left(y_{h}-y\left(u_{h}\right)\right)(\tau), v(t)\right) d \tau .
\end{aligned}
$$

From (2.22) and (3.4), we obtain

$$
\begin{gathered}
\left(\omega_{h}, \frac{\partial}{\partial t}\left(y_{h}-y\left(u_{h}\right)\right)\right)+a\left(\frac{\partial}{\partial t}\left(y_{h}-y\left(u_{h}\right)\right), \omega_{h}\right)+d\left(y_{h}-y\left(u_{h}\right), \omega_{h}\right) \\
\quad+\int_{0}^{t} c\left(t, \tau ;\left(y_{h}-y\left(u_{h}\right)\right)(\tau), \omega_{h}(t)\right) d \tau=0, \quad \forall \omega_{h} \in V^{h}
\end{gathered}
$$

We have

$$
\begin{aligned}
& \left\langle Q\left(u_{h}\right), v\right\rangle \\
& =\left(\frac{\partial}{\partial t}\left(y_{h}-y\left(u_{h}\right)\right), v-\pi_{h} v\right)+a\left(\frac{\partial}{\partial t}\left(y_{h}-y\left(u_{h}\right)\right), v-\pi_{h} v\right)+d\left(y_{h}-y\left(u_{h}\right), v-\pi_{h} v\right) \\
& \quad+\int_{0}^{t} c\left(t, \tau ;\left(y_{h}-y\left(u_{h}\right)\right)(\tau),\left(v-\pi_{h} v\right)(t)\right) d \tau \\
& =\left(\frac{\partial y_{h}}{\partial t}, v-\pi_{h} v\right)+a\left(\frac{\partial y_{h}}{\partial t}, v-\pi_{h} v\right)+d\left(y_{h}, v-\pi_{h} v\right) \\
& \quad+\int_{0}^{t} c\left(t, \tau ; y_{h}(\tau),\left(v-\pi_{h} v\right)(t)\right) d \tau-\left(f+B u_{h}, v-\pi_{h} v\right) \\
& =\sum_{\tau} \int_{\tau}\left(\frac{\partial y_{h}}{\partial t}-\operatorname{div}\left(A \nabla \frac{\partial y_{h}}{\partial t}\right)-\operatorname{div}\left(D \nabla y_{h}\right)-\int_{0}^{t} \operatorname{div}\left(C(t, \tau) \nabla y_{h}\right) d \tau-f-B u_{h}\right)\left(v-\pi_{h} v\right)
\end{aligned}
$$


Abstract and Applied Analysis

$$
\begin{aligned}
& +\sum_{\tau} \int_{\partial \tau}\left[\left(A \nabla \frac{\partial y_{h}}{\partial t}\right) \cdot n+\left(D \nabla y_{h}\right) \cdot n+\int_{0}^{t}\left(C(t, \tau) \nabla y_{h}\right) \cdot n d \tau\right]\left(v-\pi_{h} v\right) \\
\leqslant & \left\{\sum_{\tau} \int_{\tau} h_{\tau}^{2}\left(\frac{\partial}{\partial t} y_{h}-\operatorname{div}\left(A \nabla \frac{\partial y_{h}}{\partial t}\right)-\operatorname{div}\left(D \nabla y_{h}\right)-\int_{0}^{t} \operatorname{div}\left(C(t, \tau) \nabla y_{h}\right) d \tau-f-B u_{h}\right)^{2}\right. \\
& \left.+\sum_{\tau} \int_{\partial \tau} h_{l}\left[\left(A \nabla \frac{\partial y_{h}}{\partial t}\right) \cdot n+\left(D \nabla y_{h}\right) \cdot n+\int_{0}^{t}\left(C(t, \tau) \nabla y_{h}\right) \cdot n d \tau\right]^{2}\right\}^{1 / 2}\|v\|_{1, \Omega} .
\end{aligned}
$$

By setting $v=y_{h}-y\left(u_{h}\right)$ and Swartz inequality, we have

$$
\begin{aligned}
& \frac{1}{2} \frac{d}{d t}\left\|y_{h}-y\left(u_{h}\right)\right\|_{0, \Omega}^{2}+\frac{1}{2} \frac{d}{d t} a\left(y_{h}-y\left(u_{h}\right), y_{h}-y\left(u_{h}\right)\right)+c\left\|y_{h}-y\left(u_{h}\right)\right\|_{1, \Omega}^{2} \\
& \leqslant \sum_{\tau} \int_{\tau} h_{\tau}^{2}\left(\frac{\partial y_{h}}{\partial t}-\operatorname{div}\left(A \nabla \frac{\partial y_{h}}{\partial t}\right)-\operatorname{div}\left(D \nabla y_{h}\right)-\int_{0}^{t} \operatorname{div}\left(C(t, \tau) \nabla y_{h}\right) d \tau-f-B u_{h}\right)^{2} \\
& \quad+\sum_{\tau} \int_{\partial \tau} h_{l}\left[\left(A \nabla \frac{\partial y_{h}}{\partial t}\right) \cdot n+\left(D \nabla y_{h}\right) \cdot n+\int_{0}^{t}\left(C(t, \tau) \nabla y_{h}\right) \cdot n d \tau\right]^{2} \\
& \quad+\delta\left\|y_{h}-y\left(u_{h}\right)\right\|_{1, \Omega}^{2}-\int_{0}^{t} c\left(t, \tau ;\left(y_{h}-y\left(u_{h}\right)\right)(\tau),\left(y_{h}-y\left(u_{h}\right)\right)(t)\right) d \tau .
\end{aligned}
$$

Integrating time from 0 to $t$ in (3.38), we obtain

$$
\begin{aligned}
& \left\|y_{h}-y\left(u_{h}\right)\right\|_{1, \Omega}^{2}+c \int_{0}^{t}\left\|y_{h}-y\left(u_{h}\right)\right\|_{1, \Omega}^{2} d \tau \\
& \leqslant C\left\{\int _ { 0 } ^ { t } \sum _ { \tau } h _ { \tau } ^ { 2 } \int _ { \tau } \left(\frac{\partial y_{h}}{\partial t}-\operatorname{div}\left(A \nabla \frac{\partial y_{h}}{\partial t}\right)-\operatorname{div}\left(D \nabla y_{h}\right)\right.\right. \\
& \left.\quad-\int_{0}^{\tau} \operatorname{div}\left(C(\tau, s) \nabla y_{h}\right) d s-f-B u_{h}\right) d \tau \\
& \left.+\int_{0}^{t} \sum_{\tau} h_{l} \int_{\partial \tau}\left[\left(A \nabla \frac{\partial y_{h}}{\partial t}\right) \cdot n+\left(D \nabla y_{h}\right) \cdot n+\int_{0}^{\tau}\left(C(\tau, s) \nabla y_{h}\right) \cdot n d s\right]^{2} d t\right\} \\
& +\delta \int_{0}^{t}\left\|y_{h}-y\left(u_{h}\right)\right\|_{1, \Omega}^{2} d \tau+C \int_{0}^{t} \int_{0}^{\tau}\left\|y_{h}-y\left(u_{h}\right)\right\|_{1, \Omega}^{2} d s d \tau+C\left\|y_{0}-y_{0}^{h}\right\|_{1, \Omega}^{2} .
\end{aligned}
$$

Since $\delta$ is small enough, then from (3.39) and Gronwall inequality, we have

$$
\int_{0}^{t}\left\|y_{h}-y\left(u_{h}\right)\right\|_{1, \Omega}^{2} d t
$$




$$
\begin{aligned}
& \leqslant C \int_{0}^{t} \sum_{\tau} h_{\tau}^{2} \int_{\tau}\left(\frac{\partial y_{h}}{\partial t}-\operatorname{div}\left(A \nabla \frac{\partial y_{h}}{\partial t}\right)-\operatorname{div}\left(D \nabla y_{h}\right)\right. \\
& \left.\quad-\int_{0}^{\tau} \operatorname{div}\left(C(\tau, s) \nabla y_{h}\right) d s-f-B u_{h}\right)^{2} d \tau \\
& +C \int_{0}^{t} \sum_{\tau} h_{l} \int_{\partial \tau}\left[\left(A \nabla \frac{\partial y_{h}}{\partial t}\right) \cdot n+\left(D \nabla y_{h}\right) \cdot n+\int_{0}^{\tau}\left(C(\tau, s) \nabla y_{h}\right) \cdot n d s\right]^{2} d t+C\left\|y_{0}-y_{0}^{h}\right\|_{1, \Omega}^{2} .
\end{aligned}
$$

Then,

$$
\begin{aligned}
& \left\|y_{h}-y\left(u_{h}\right)\right\|_{L^{2}\left(0, T ; H^{1}(\Omega)\right)}^{2} \leqslant C\left(\eta_{4}^{2}+\eta_{5}^{2}+\eta_{6}^{2}\right), \\
& \left\|y_{h}-y\left(u_{h}\right)\right\|_{L^{\infty}\left(0, T ; H^{1}(\Omega)\right)}^{2} \leqslant C\left(\eta_{4}^{2}+\eta_{5}^{2}+\eta_{6}^{2}\right) .
\end{aligned}
$$

In the same way of getting (3.34), we can similarly obtain

$$
\left\|\frac{\partial}{\partial t}\left(y_{h}-y\left(u_{h}\right)\right)\right\|_{L^{2}\left(0, T ; H^{1}(\Omega)\right)}^{2} \leqslant C\left(\eta_{4}^{2}+\eta_{5}^{2}+\eta_{6}^{2}\right) .
$$

Then the desired results (3.23) follow from (3.32)-(3.34) and (3.41)-(3.42).

From Lemmas 3.1 and 3.2, we have the following results.

Theorem 3.3. Let $(y, p, u)$ and $\left(y_{h}, p_{h}, u_{h}\right)$ be the solutions of $(2.17)-(2.19)$ and $(2.22)-(2.24)$ respectively. Then, there hold the a posteriori error estimates as

$$
\begin{aligned}
& \left\|u-u_{h}\right\|_{L^{2}\left(0, T ; L^{2}\left(\Omega_{U}\right)\right)}^{2}+\left\|y-y_{h}\right\|_{L^{\infty}\left(0, T ; H^{1}(\Omega)\right)}^{2}+\left\|\frac{\partial}{\partial t}\left(y-y_{h}\right)\right\|_{L^{2}\left(0, T ; H^{1}(\Omega)\right)}^{2} \\
& \quad+\left\|p-p_{h}\right\|_{L^{\infty}\left(0, T ; H^{1}(\Omega)\right)}^{2}+\left\|\frac{\partial}{\partial t}\left(p-p_{h}\right)\right\|_{L^{2}\left(0, T ; H^{1}(\Omega)\right)}^{2} \leqslant C \sum_{i=1}^{6} \eta_{i}^{2},
\end{aligned}
$$

where $\eta_{1}^{2}$ is defined in Lemma 3.1.

Proof. First, from (3.27) and (3.36), and [2], we have the following stability results:

$$
\begin{aligned}
\left\|y-y\left(u_{h}\right)\right\|_{L^{\infty}\left(0, T ; H^{1}(\Omega)\right)}^{2}+\left\|\frac{\partial}{\partial t}\left(y-y\left(u_{h}\right)\right)\right\|_{L^{2}\left(0, T ; H^{1}(\Omega)\right)}^{2} & \leqslant C\left\|u-u_{h}\right\|_{L^{2}\left(0, T ; L^{2}(\Omega U)\right)^{\prime}}^{2} \\
\left\|p-p\left(u_{h}\right)\right\|_{L^{\infty}\left(0, T ; H^{1}(\Omega)\right)}^{2}+\left\|\frac{\partial}{\partial t}\left(p-p\left(u_{h}\right)\right)\right\|_{L^{2}\left(0, T ; H^{1}(\Omega)\right)}^{2} & \leqslant C\left\|y-y\left(u_{h}\right)\right\|_{L^{2}\left(0, T ; L^{2}(\Omega)\right)}^{2} \\
& \leqslant C\left\|u-u_{h}\right\|_{L^{2}\left(0, T ; L^{2}\left(\Omega_{U}\right)\right)}^{2} .
\end{aligned}
$$


Then, the desired results (3.43) follows from triangle inequality, (3.44) and Lemmas 3.1 and 3.2.

This completes the proof.

\section{A Posteriori Error Estimates in $L^{2}\left(0, T ; L^{2}(\Omega)\right)$-Norm}

In the following, we will derive the a posteriori error estimates in $L^{2}\left(0, T ; L^{2}(\Omega)\right)$-norm.

For given $F \in L^{2}\left(0, T ; L^{2}(\Omega)\right)$, we have

$$
\begin{gathered}
\frac{\partial \phi}{\partial t}-\operatorname{div}\left(A \nabla \frac{\partial \phi}{\partial t}\right)-\operatorname{div}(D \nabla \phi)-\int_{0}^{t} \operatorname{div}(C(t, \tau) \nabla \phi(\tau)) d \tau=F, \quad(x, t) \in \Omega \times(0, T] \\
\frac{\phi}{\partial \Omega}=0, \quad t \in(0, T] \\
\phi(x, 0)=0, \quad x \in \Omega,
\end{gathered}
$$

and its dual equation

$$
\begin{gathered}
-\frac{\partial \psi}{\partial t}+\operatorname{div}\left(A^{*} \nabla \frac{\partial \psi}{\partial t}\right)-\operatorname{div}\left(D^{*} \nabla \psi\right)-\int_{t}^{T} \operatorname{div}\left(C^{*}(\tau, t) \nabla \psi(\tau)\right) d \tau=F, \quad(x, t) \in \Omega \times(0, T], \\
\frac{\psi}{\partial \Omega}=0, \quad t \in(0, T] \\
\psi(x, T)=0, \quad x \in \Omega .
\end{gathered}
$$

From $[1,2]$, we have the following stability results.

Lemma 4.1. Assume that $\Omega$ is a convex domain. Let $\phi$ and $\psi$ be the solution of (4.1) and (4.2), respectively. Then,

$$
\begin{aligned}
\|\phi\|_{L^{\infty}\left(0, T ; L^{2}(\Omega)\right)} & \leqslant C\|F\|_{L^{2}\left(0, T ; L^{2}(\Omega)\right)} \\
\|\nabla \phi\|_{L^{2}\left(0, T ; L^{2}(\Omega)\right)} & \leqslant C\|F\|_{L^{2}\left(0, T ; L^{2}(\Omega)\right)} \\
\left\|D^{2} \phi\right\|_{L^{2}\left(0, T ; L^{2}(\Omega)\right)} & \leqslant C\|F\|_{L^{2}\left(0, T ; L^{2}(\Omega)\right)} \\
\left\|\frac{\partial}{\partial t} \phi\right\|_{L^{2}\left(0, T ; L^{2}(\Omega)\right)} & \leqslant C\|F\|_{L^{2}\left(0, T ; L^{2}(\Omega)\right)} \\
\|\psi\|_{L^{\infty}\left(0, T ; L^{2}(\Omega)\right)} & \leqslant C\|F\|_{L^{2}\left(0, T ; L^{2}(\Omega)\right)} \\
\|\nabla \psi\|_{L^{2}\left(0, T ; L^{2}(\Omega)\right)} & \leqslant C\|F\|_{L^{2}\left(0, T ; L^{2}(\Omega)\right)} \\
\left\|D^{2} \psi\right\|_{L^{2}\left(0, T ; L^{2}(\Omega)\right)} & \leqslant C\|F\|_{L^{2}\left(0, T ; L^{2}(\Omega)\right)} \\
\left\|\frac{\partial}{\partial t} \psi\right\|_{L^{2}\left(0, T ; L^{2}(\Omega)\right)} & \leqslant C\|F\|_{L^{2}\left(0, T ; L^{2}(\Omega)\right)}
\end{aligned}
$$

where $D^{2} \phi=\partial^{2} \phi / \partial x_{i} \partial x_{j}, 1 \leqslant i, j \leqslant n$, and $D^{2} \psi$ is defined similarly. 
Using Lemmas 3.1 and 4.1, we have the following upperbounds.

Lemma 4.2. Let $(y, p, u)$ and $\left(y_{h}, p_{h}, u_{h}\right)$ be the solutions of (2.17)-(2.19) and (2.22)-(2.24), respectively. Then, there hold the a posteriori error estimates as

$$
\left\|y_{h}-y\left(u_{h}\right)\right\|_{L^{2}\left(0, T ; L^{2}(\Omega)\right)}^{2}+\left\|p_{h}-p\left(u_{h}\right)\right\|_{L^{2}\left(0, T ; L^{2}(\Omega)\right)}^{2} \leqslant C\left(\sum_{i=2}^{5} \xi_{i}^{2}+\eta_{6}^{2}\right)
$$

where $\eta_{6}^{2}$ is defined in Lemma 3.2, and

$$
\begin{gathered}
\xi_{2}^{2}=\int_{0}^{T}\left\{\sum _ { \tau } h _ { \tau } ^ { 4 } \int _ { \tau } \left(\frac{\partial p_{h}}{\partial t}-\operatorname{div}\left(A^{*} \nabla \frac{\partial p_{h}}{\partial t}\right)+\operatorname{div}\left(D^{*} \nabla p_{h}\right)\right.\right. \\
\left.\left.\quad+\int_{t}^{T} \operatorname{div}\left(C^{*}(\tau, t) \nabla p_{h}(\tau)\right) d \tau+y_{h}-z_{d}\right)^{2}\right\} d t, \\
\xi_{3}^{2}=\int_{0}^{T} \sum_{\tau} h_{l}^{3} \int_{\partial \tau}\left[-\left(A^{*} \nabla \frac{\partial p_{h}}{\partial t}\right) \cdot n+\left(D^{*} \nabla p_{h}\right) \cdot n+\int_{t}^{T}\left(C^{*}(\tau, t) \nabla p_{h}(\tau)\right) \cdot n d \tau\right]^{2} d l d t, \\
\xi_{4}^{2}=\int_{0}^{T}\left\{\sum _ { \tau } h _ { \tau } ^ { 4 } \int _ { \tau } \left(\frac{\partial y_{h}}{\partial t}-\operatorname{div}\left(A \nabla \frac{\partial y_{h}}{\partial t}\right)-\operatorname{div}\left(D \nabla y_{h}\right)\right.\right. \\
\left.\left.-\int_{0}^{t} \operatorname{div}\left(C(t, \tau) \nabla y_{h}(\tau)\right) d \tau-f-B u_{h}\right)^{2}\right\} d t, \\
\xi_{5}^{2}=\int_{0}^{T} \sum_{\tau} h_{l}^{3} \int_{\partial \tau}\left[\left(A \nabla \frac{\partial y_{h}}{\partial t}\right) \cdot n+\left(D \nabla y_{h}\right) \cdot n+\int_{0}^{t}\left(C(t, \tau) \nabla y_{h}(\tau)\right) \cdot n d \tau\right]^{2} d l d t .
\end{gathered}
$$

Proof. We first estimate $\left\|p_{h}-p\left(u_{h}\right)\right\|_{L^{2}\left(0, T ; L^{2}(\Omega)\right)}^{2}$.

Let $\phi$ be the solution of (4.1) with $F=p_{h}-p\left(u_{h}\right)$, and $\phi_{I}=\widehat{\pi}_{h} \phi$ the interpolation of $\phi$ in Lemma 2.3.

From (4.1), (3.27), and by integrating by parts we obtain

$$
\begin{aligned}
& \left\|p_{h}-p\left(u_{h}\right)\right\|_{L^{2}\left(0, T ; L^{2}(\Omega)\right)}^{2} \\
& =\int_{0}^{T}\left(F(t),\left(p_{h}-p\left(u_{h}\right)\right)(t)\right) d t \\
& =\int_{0}^{T}\left[-\left(\frac{\partial}{\partial t}\left(p_{h}-p\left(u_{h}\right)\right), \phi\right)-a\left(\phi, \frac{\partial}{\partial t}\left(p_{h}-p\left(u_{h}\right)\right)\right)\right. \\
& \left.\quad+d\left(\phi, p_{h}-p\left(u_{h}\right)\right)+\int_{0}^{t} c\left(t, \tau ; \phi(\tau),\left(p_{h}-p\left(u_{h}\right)\right)(t)\right) d \tau\right] d t
\end{aligned}
$$




$$
\begin{aligned}
& =\int_{0}^{T}\left[-\left(\frac{\partial}{\partial t}\left(p_{h}-p\left(u_{h}\right)\right), \phi-\phi_{I}\right)-a\left(\phi-\phi_{I}, \frac{\partial}{\partial t}\left(p_{h}-p\left(u_{h}\right)\right)\right)\right. \\
& +d\left(\phi-\phi_{I}, p_{h}-p\left(u_{h}\right)\right)+\int_{0}^{t} c\left(t, \tau ;\left(\phi-\phi_{I}\right)(\tau),\left(p_{h}-p\left(u_{h}\right)\right)(t)\right) d \tau \\
& -\left(\frac{\partial}{\partial t}\left(p_{h}-p\left(u_{h}\right)\right), \phi_{I}\right)-a\left(\phi_{I}, \frac{\partial}{\partial t}\left(p_{h}-p\left(u_{h}\right)\right)\right) \\
& \left.+d\left(\phi_{I}, p_{h}-p\left(u_{h}\right)\right)+\int_{0}^{t} c\left(t, \tau ; \phi_{I}(\tau),\left(p_{h}-p\left(u_{h}\right)\right)(t)\right) d \tau\right] d t \\
& =\int_{0}^{T}\left[-\left(\frac{\partial p_{h}}{\partial t}, \phi-\phi_{I}\right)-a\left(\phi-\phi_{I}, \frac{\partial p_{h}}{\partial t}\right)\right. \\
& +d\left(\phi-\phi_{I}, p_{h}\right)+\int_{0}^{t} c\left(t, \tau ;\left(\phi-\phi_{I}\right)(\tau), p_{h}(t)\right) d \tau+\left(\frac{\partial p\left(u_{h}\right)}{\partial t}, \phi-\phi_{I}\right) \\
& +a\left(\phi-\phi_{I}, \frac{\partial p\left(u_{h}\right)}{\partial t}\right)-d\left(\phi-\phi_{I}, p\left(u_{h}\right)\right)-\int_{0}^{t} c\left(t, \tau ;\left(\phi-\phi_{I}\right)(\tau), p\left(u_{h}\right)(t)\right) d \tau \\
& -\left(\frac{\partial}{\partial t}\left(p_{h}-p\left(u_{h}\right)\right), \phi_{I}\right)-a\left(\phi_{I}, \frac{\partial}{\partial t}\left(p_{h}-p\left(u_{h}\right)\right)\right) \\
& \left.+d\left(\phi_{I}, p_{h}-p\left(u_{h}\right)\right)+\int_{0}^{t} c\left(t, \tau ; \phi_{I}(\tau),\left(p_{h}-p\left(u_{h}\right)\right)(t)\right) d \tau\right] d t \\
& =\int_{0}^{T}\left[-\left(\frac{\partial p_{h}}{\partial t}, \phi-\phi_{I}\right)-a\left(\phi-\phi_{I}, \frac{\partial p_{h}}{\partial t}\right)\right. \\
& +d\left(\phi-\phi_{I}, p_{h}\right)+\int_{t}^{T} c\left(\tau, t ;\left(\phi-\phi_{I}\right)(t), p_{h}(\tau)\right) d \tau \\
& +\left(\frac{\partial p\left(u_{h}\right)}{\partial t}, \phi-\phi_{I}\right)+a\left(\phi-\phi_{I}, \frac{\partial p\left(u_{h}\right)}{\partial t}\right) \\
& -d\left(\phi-\phi_{I}, p\left(u_{h}\right)\right)-\int_{t}^{T} c\left(\tau, t ;\left(\phi-\phi_{I}\right)(t), p\left(u_{h}\right)(\tau)\right) d \tau \\
& -\left(\frac{\partial}{\partial t}\left(p_{h}-p\left(u_{h}\right)\right), \phi_{I}\right)-a\left(\phi_{I}, \frac{\partial}{\partial t}\left(p_{h}-p\left(u_{h}\right)\right)\right) \\
& \left.+d\left(\phi_{I}, p_{h}-p\left(u_{h}\right)\right)+\int_{t}^{T} c\left(\tau, t ; \phi_{I}(t),\left(p_{h}-p\left(u_{h}\right)\right)(\tau)\right) d \tau\right] d t \\
& =\int_{0}^{T}\left[-\left(\frac{\partial p_{h}}{\partial t}, \phi-\phi_{I}\right)-a\left(\phi-\phi_{I}, \frac{\partial p_{h}}{\partial t}\right)+d\left(\phi-\phi_{I}, p_{h}\right)+\int_{t}^{T} c\left(\tau, t ;\left(\phi-\phi_{I}\right)(t), p_{h}(\tau)\right) d \tau\right] d t \\
& -\int_{0}^{T}\left(y\left(u_{h}\right)-z_{d}, \phi-\phi_{I}\right) d t+\int_{0}^{T}\left(y_{h}-y\left(u_{h}\right), \phi_{I}\right) d t \\
& =\int_{0}^{T}\left\{\sum _ { \tau } \int _ { \tau } \left(-\frac{\partial p_{h}}{\partial t}+\operatorname{div}\left(A^{*} \nabla \frac{\partial p_{h}}{\partial t}\right)-\operatorname{div}\left(D^{*} \nabla p_{h}\right)\right.\right.
\end{aligned}
$$




$$
\begin{gathered}
\left.\left.+\int_{t}^{T} \operatorname{div}\left(C^{*}(\tau, t) \nabla p_{h}(\tau)\right) d \tau+y_{h}-z_{d}\right)\left(\phi-\phi_{I}\right)\right\} d t \\
+\int_{0}^{T} \sum_{\tau} \int_{\partial \tau}\left[-\left(A^{*} \nabla \frac{\partial p_{h}}{\partial t}\right) \cdot n+\left(D^{*} \nabla p_{h}\right) \cdot n+\int_{t}^{T}\left(C^{*}(\tau, t) \nabla p_{h}(\tau)\right) \cdot n d \tau\right] \\
\times\left(\phi-\phi_{I}\right) d l d t+\int_{0}^{T}\left(y_{h}-y\left(u_{h}\right), \phi\right) d t \\
=J_{1}+J_{2}+J_{3} .
\end{gathered}
$$

It follows from Lemmas $2.3,2.5$, and 4.1 that

$$
\begin{aligned}
J_{1} \leqslant & C(\delta) \int_{0}^{T}\left\{\sum _ { \tau } h _ { \tau } ^ { 4 } \int _ { \tau } \left(\frac{\partial p_{h}}{\partial t}-\operatorname{div}\left(A^{*} \nabla \frac{\partial p_{h}}{\partial t}\right)+\operatorname{div}\left(D^{*} \nabla p_{h}\right)\right.\right. \\
& \left.\left.\quad+\int_{t}^{T} \operatorname{div}\left(C^{*}(\tau, t) \nabla p_{h}(\tau)\right) d \tau+y_{h}-z_{d}\right)^{2}\right\} d t \\
& +\delta \int_{0}^{T}|\phi|_{2, \Omega}^{2} d t \leqslant C(\delta) \xi_{2}^{2}+\delta\left\|p_{h}-p\left(u_{h}\right)\right\|_{L^{2}\left(0, T ; L^{2}(\Omega)\right)^{\prime}}^{2} \\
J_{2} \leqslant & C(\delta) \int_{0}^{T} \sum_{\tau} h_{l}^{3} \int_{\partial \tau}\left[-\left(A^{*} \nabla \frac{\partial p_{h}}{\partial t}\right) \cdot n+\left(D^{*} \nabla p_{h}\right) \cdot n+\int_{t}^{T}\left(C^{*}(\tau, t) \nabla p_{h}(\tau)\right) \cdot n d \tau\right]^{2} d l d t \\
& +\delta \int_{0}^{T}|\phi|_{0, \Omega}^{2} d t \\
\leqslant & C(\delta) \xi_{3}^{2}+\delta\left\|p_{h}-p\left(u_{h}\right)\right\|_{L^{2}\left(0, T ; L^{2}(\Omega)\right)}^{2}
\end{aligned}
$$

By Schwartz inequality, we have

$$
J_{3} \leqslant C(\delta)\left\|y_{h}-y\left(u_{h}\right)\right\|_{L^{2}\left(0, T ; L^{2}(\Omega)\right)}^{2}+\delta\left\|p_{h}-p\left(u_{h}\right)\right\|_{L^{2}\left(0, T ; L^{2}(\Omega)\right)}^{2}
$$

Letting $\delta$ be small enough, it follows from (4.6)-(4.8) that

$$
\left\|p_{h}-p\left(u_{h}\right)\right\|_{L^{2}\left(0, T ; L^{2}(\Omega)\right)}^{2} \leqslant C \sum_{i=2}^{3} \xi_{i}^{2}+C\left\|y_{h}-y\left(u_{h}\right)\right\|_{L^{2}\left(0, T ; L^{2}(\Omega)\right)}^{2} .
$$

Next, we estimate $\left\|y_{h}-y\left(u_{h}\right)\right\|_{L^{2}\left(0, T ; L^{2}(\Omega)\right)}^{2}$. Similarly let $\psi$ be the solution of (4.2) with $F=$ $y_{h}-y\left(u_{h}\right)$, and $\psi_{I}=\widehat{\pi}_{h} \psi$ the interpolation of $\psi$ in Lemma 2.3. 
Then, it follows from (3.36) and integrating by parts that

$$
\begin{aligned}
& \left\|y_{h}-y\left(u_{h}\right)\right\|_{L^{2}\left(0, T ; L^{2}(\Omega)\right)}^{2} \\
& =\int_{0}^{T}\left(F(t),\left(y_{h}-y\left(u_{h}\right)\right)(t)\right) d t \\
& =\int_{0}^{T}\left[\left(\frac{\partial}{\partial t}\left(y_{h}-y\left(u_{h}\right)\right), \psi\right)+a\left(\frac{\partial}{\partial t}\left(y_{h}-y\left(u_{h}\right)\right), \psi\right)+d\left(y_{h}-y\left(u_{h}\right), \psi\right)\right. \\
& \left.+\int_{t}^{T} c\left(\tau, t ;\left(y_{h}-y\left(u_{h}\right)\right)(t), \psi(\tau)\right) d \tau\right] d t \\
& +a\left(y_{0}^{h}-y_{0}, \psi(0)\right)+\left(y_{0}^{h}-y_{0}, \psi(0)\right) \\
& =\int_{0}^{T}\left[\left(\frac{\partial}{\partial t}\left(y_{h}-y\left(u_{h}\right)\right), \psi-\psi_{I}\right)+a\left(\frac{\partial}{\partial t}\left(y_{h}-y\left(u_{h}\right)\right), \psi-\psi_{I}\right)\right. \\
& \left.+d\left(y_{h}-y\left(u_{h}\right), \psi-\psi_{I}\right)+\int_{t}^{T} c\left(\tau, t ;\left(y_{h}-y\left(u_{h}\right)\right)(t),\left(\psi-\psi_{I}\right)(\tau)\right) d \tau\right] d t \\
& +\int_{0}^{T}\left[\left(\frac{\partial}{\partial t}\left(y_{h}-y\left(u_{h}\right)\right), \psi_{I}\right)+a\left(\frac{\partial}{\partial t}\left(y_{h}-y\left(u_{h}\right)\right), \psi_{I}\right)\right. \\
& \left.+d\left(y_{h}-y\left(u_{h}\right), \psi_{I}\right)+\int_{t}^{T} c\left(\tau, t ;\left(y_{h}-y\left(u_{h}\right)\right)(t), \psi_{I}(\tau)\right) d \tau\right] d t \\
& +a\left(y_{0}^{h}-y_{0}, \psi(0)\right)+\left(y_{0}^{h}-y_{0}, \psi(0)\right) \\
& =\int_{0}^{T}\left[\left(\frac{\partial y_{h}}{\partial t}, \psi-\psi_{I}\right)+a\left(\frac{\partial y_{h}}{\partial t}, \psi-\psi_{I}\right)+d\left(y_{h}, \psi-\psi_{I}\right)\right. \\
& \left.+\int_{0}^{t} c\left(t, \tau ; y_{h}(\tau),\left(\psi-\psi_{I}\right)(t)\right) d \tau\right] d t \\
& -\int_{0}^{T}\left(f+B u_{h}, \psi-\psi_{I}\right) d t+a\left(y_{0}^{h}-y_{0}, \psi(0)\right)+\left(y_{0}^{h}-y_{0}, \psi(0)\right) \\
& =\int_{0}^{T}\left\{\sum _ { \tau } \int _ { \tau } \left(\frac{\partial y_{h}}{\partial t}-\operatorname{div}\left(A \nabla \frac{\partial y_{h}}{\partial t}\right)-\operatorname{div}\left(D \nabla y_{h}\right)\right.\right. \\
& \left.\left.-\int_{0}^{t} \operatorname{div}\left(C(\tau, t) \nabla y_{h}(\tau)\right) d \tau-f-B u_{h}\right)\left(\psi-\psi_{I}\right)\right\} d t \\
& +\int_{0}^{T} \sum_{\tau} \int_{\partial \tau}\left[\left(A \nabla \frac{\partial y_{h}}{\partial t}\right) \cdot n+\left(D \nabla y_{h}\right) \cdot n-\int_{0}^{t}\left(C(t, \tau) \nabla y_{h}(\tau)\right) \cdot n\right] d \tau\left(\psi-\psi_{I}\right) d l d t \\
& +a\left(y_{0}^{h}-y_{0}, \psi(0)\right)+\left(y_{0}^{h}-y_{0}, \psi(0)\right)=D_{1}+D_{2}+D_{3}+D_{4} \text {. }
\end{aligned}
$$


Similarly, it follows from Lemmas 2.3, 2.5 and 4.1 that

$$
\begin{aligned}
& D_{1} \leqslant C(\delta) \int_{0}^{T}\left\{\sum _ { \tau } h _ { \tau } ^ { 4 } \int _ { \tau } \left(\frac{\partial y_{h}}{\partial t}-\operatorname{div}\left(A \nabla \frac{\partial y_{h}}{\partial t}\right)-\operatorname{div}\left(D \nabla y_{h}\right)\right.\right. \\
&\left.\left.\quad-\int_{0}^{t} \operatorname{div}\left(C(\tau, t) \nabla y_{h}(\tau)\right) d \tau-f-B u_{h}\right)^{2}\right\} d t \\
&+\delta \int_{0}^{T}|\psi|_{2, \Omega}^{2} d t \leqslant C \xi_{4}^{2}+\delta\left\|y_{h}-y\left(u_{h}\right)\right\|_{L^{2}\left(0, T ; L^{2}(\Omega)\right)^{\prime}}^{2} \\
& D_{2} \leqslant C(\delta) \int_{0}^{T} \sum_{\tau} h_{l}^{3} \int_{\partial \tau}\left[\left(A \nabla \frac{\partial y_{h}}{\partial t}\right) \cdot n+\left(D \nabla y_{h}\right) \cdot n-\int_{0}^{t}\left(C(t, \tau) \nabla y_{h}(\tau)\right) \cdot n\right]^{2} d l d t \\
&+\delta \int_{0}^{T}|\psi|_{2, \Omega}^{2} d t \leqslant C \xi_{5}^{2}+\delta\left\|y_{h}-y\left(u_{h}\right)\right\|_{L^{2}\left(0, T ; L^{2}(\Omega)\right)^{\prime}}^{2} \\
& D_{3}+D_{4} \leqslant C \eta_{6}^{2}+\delta\left\|y_{h}-y\left(u_{h}\right)\right\|_{L^{2}\left(0, T ; L^{2}(\Omega)\right)}^{2}
\end{aligned}
$$

Letting $\delta$ be small enough, then from (4.10)-(4.11), we have

$$
\left\|y_{h}-y\left(u_{h}\right)\right\|_{L^{2}\left(0, T ; L^{2}(\Omega)\right)}^{2} \leqslant C\left(\xi_{4}^{2}+\xi_{5}^{2}+\eta_{6}^{2}\right)
$$

The desired results (4.4) follows from (4.9)-(4.12).This completes the proof.

Using Lemmas 3.1 and 4.2, we have the following upper bounds.

Theorem 4.3. Let $(y, p, u)$ and $\left(y_{h}, p_{h}, u_{h}\right)$ be the solutions of (2.17)-(2.19) and (2.22)-(2.24), respectively. Then, there hold the a posteriori error estimates as

$$
\left\|u-u_{h}\right\|_{L^{2}\left(0, T ; L^{2}\left(\Omega_{U}\right)\right)}^{2}+\left\|y-y_{h}\right\|_{L^{2}\left(0, T ; L^{2}(\Omega)\right)}^{2}+\left\|p-p_{h}\right\|_{L^{2}\left(0, T ; L^{2}(\Omega)\right)}^{2} \leqslant C\left(\eta_{1}^{2}+\sum_{i=2}^{5} \xi_{i}^{2}+\eta_{6}^{2}\right) .
$$

Proof. By triangle inequality, (3.44) and (3.38), Lemmas 3.1 and 4.2, we can easily prove (4.13) in the same way of getting (3.43).

This completes the proof.

\section{Conclusion}

In this paper, we study the semi-discrete adaptive finite element method for optimal control problem governed by a linear quasiparabolic Integrodifferential equation. We extend the existing methods in studying adaptive finite element approximation of optimal control governed by a parabolic Integrodifferential equation to the control governed by a quasiparabolic Integrodifferential equation. After presenting the weak form and the existence and 
uniqueness of the solution for the optimal control problem, the a posteriori error estimates for semi-discrete finite element approximations in $L^{\infty}\left(0, T ; H^{1}(\Omega)\right)$-norm and $L^{2}\left(0, T ; L^{2}(\Omega)\right)$ norm are derived. The work will pave a way to derive the a posteriori error estimates of full discrete finite element approximations of this optimal control problem

\section{Acknowledgments}

This research was supported by Science and Technology Development Planning project of Shandong Province (no. 2011GGH20118), Shandong Province Natural Science Foundation (no. ZR2009AQ004), and National Natural Science Foundation of China (no. 11071141).

\section{References}

[1] S. B. Cui, "Global solutions for a class of nonlinear integro-differential equations," Acta Mathematicae Applicatae Sinica, vol. 16, no. 2, pp. 191-200, 1993.

[2] X. Cui, "Sobolev-Volterra projection and numerical analysis of finite element methods for integrodifferential equations," Acta Mathematicae Applicatae Sinica, vol. 24, no. 3, pp. 441-455, 2001.

[3] W. Alt, "On the approximation of infinite optimization problems with an application to optimal control problems," Applied Mathematics and Optimization, vol. 12, no. 1, pp. 15-27, 1984.

[4] R. S. Falk, "Approximation of a class of optimal control problems with order of convergence estimates," Journal of Mathematical Analysis and Applications, vol. 44, pp. 28-47, 1973.

[5] D. A. French and J. T. King, "Approximation of an elliptic control problem by the finite element method," Numerical Functional Analysis and Optimization, vol. 12, no. 3-4, pp. 299-314, 1991.

[6] K. Malanowski, "Convergence of approximations vs. regularity of solutions for convex, controlconstrained optimal-control problems," Applied Mathematics and Optimization, vol. 8, no. 1, pp. 69-95, 1982.

[7] P. Neittaanmaki, D. Tiba, and M. Dekker, Optimal Control of Nonlinear Parabolic Systems, Theory, Algorithms, and Applications, Marcel Dekker, New York, NY, USA, 1994.

[8] O. Pironneau, Optimal Shape Design for Elliptic Systems, Springer-Verlag, New York, NY, USA, 1984.

[9] D. Tiba, Lectures on the Optimal Control of Elliptic Equations, University of Jyvaskyla Press, Jyvaskyla, Finland, 1995.

[10] D. Tiba, Optimal Control of Nonsmooth Distributed Parameter Systems, vol. 1459 of Lecture Notes in Mathematics, Springer-Verlag, Berlin, Germany, 1990.

[11] D. Tiba and F. Tröltzsch, "Error estimates for the discretization of state constrained convex control problems," Numerical Functional Analysis and Optimization, vol. 17, no. 9-10, pp. 1005-1028, 1996.

[12] T. J. Sun, L. Ge, and W. B. Liu, “Equivalent a posteriori errorestimates for a constrained optimal control problem governed by parabolic equations," International Journal of Numerical Analysis and Modeling. In press.

[13] W. Liu and N. Yan, "A posteriori error estimates for optimal control problems governed by parabolic equations," Numerische Mathematik, vol. 93, no. 3, pp. 497-521, 2003.

[14] W. Liu and N. N. Yan, "A posteriori error estimates for distributed convex optimal control problems," Advances in Computational Mathematics, vol. 15, no. 1-4, pp. 285-309, 2001.

[15] W. Liu and N. Yan, "A posteriori error estimates for convex boundary control problems," SIAM Journal on Numerical Analysis, vol. 39, no. 1, pp. 73-99, 2001.

[16] B. Hermann and N. N. Yan, "Finite element methods for optimal control problems governed by integral equations and integro-differential equations," Numerische Mathematik, vol. 101, no. 1, pp. 127, 2005.

[17] J. L. Lions and E. Magenes, Non Homogeneous Boundary Value Problems and Applications, Grandlehre B., vol. 181, Springer-Verlag, New York, NY, USA, 1972.

[18] J.-L. Lions, Optimal Control of Systems Governed by Partial Differential Equations, Springer-Verlag, Berlin, Germany, 1971. 
[19] P. G. Ciarlet, The Finite Element Method for Elliptic Problems, North-Holland, Amsterdam, The Netherlands, 1978.

[20] L. R. Scott and S. Zhang, "Finite element interpolation of nonsmooth functions satisfying boundary conditions," Mathematics of Computation, vol. 54, no. 190, pp. 483-493, 1990.

[21] A. Kufner, O. John, and S. Fucik, Function Spaces, Noordhoff International Publishing, Leyden, The Netherlands, 1977. 


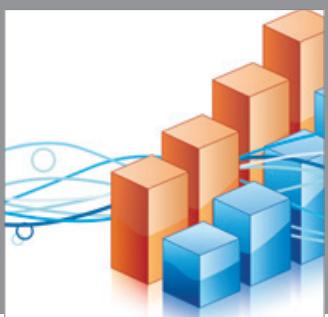

Advances in

Operations Research

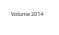

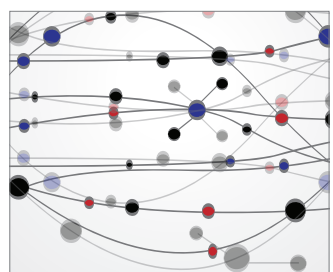

\section{The Scientific} World Journal
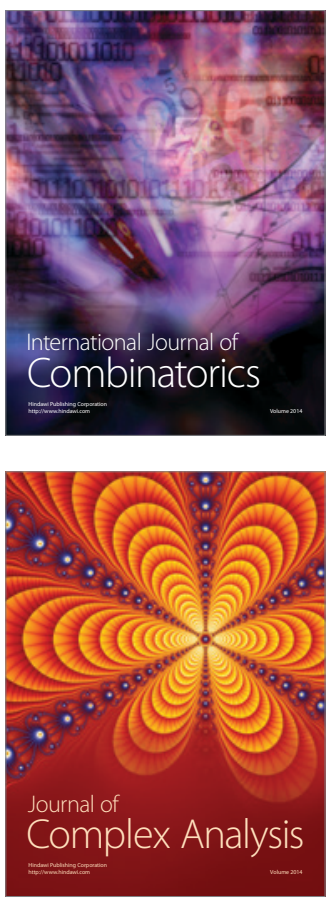

International Journal of

Mathematics and

Mathematical

Sciences
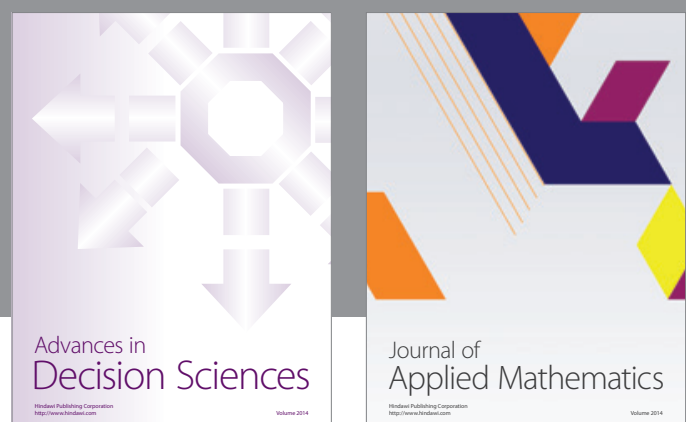

Journal of

Applied Mathematics
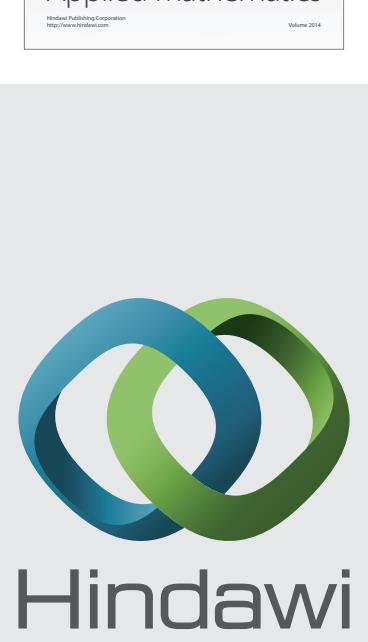

Submit your manuscripts at http://www.hindawi.com
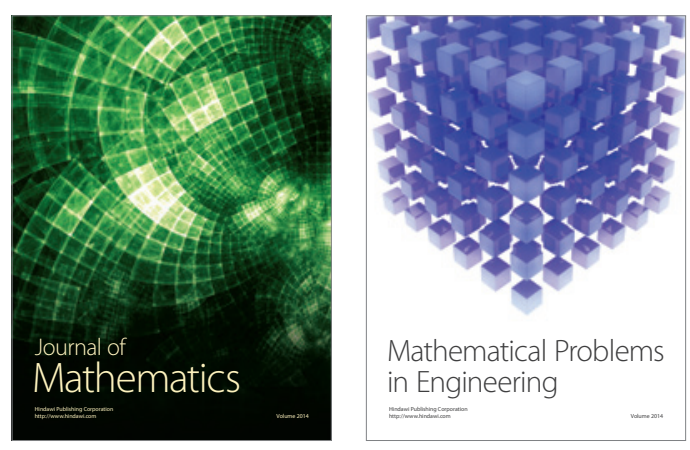

Mathematical Problems in Engineering
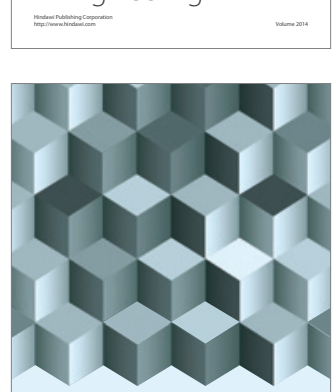

Journal of

Function Spaces
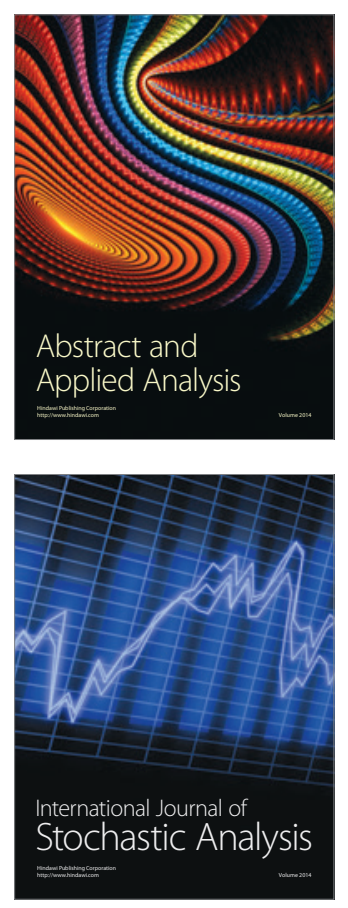

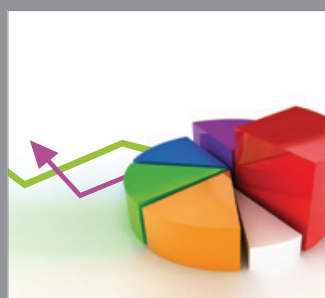

ournal of

Probability and Statistics

Promensencen
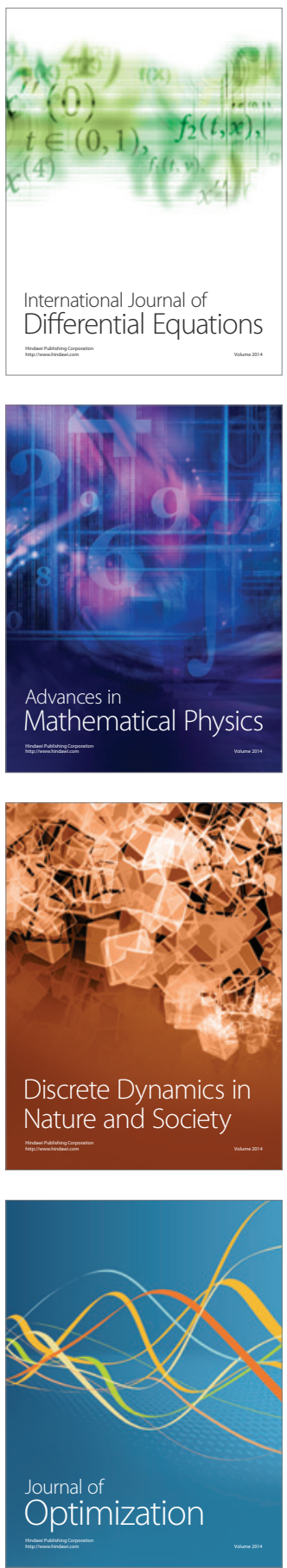